\title{
A Climatology of the Gravest Waves in the Equatorial Lower and Middle Stratosphere: Method and Results for the ERA-40 Re-Analysis and the LMDz GCM
}

\author{
FRANÇOIS LOTT, JAYANARAYANAN KUTTIPPURATH, AND FRANÇOIS VIAL \\ Laboratoire de Météorologie Dynamique du CNRS, Paris, France
}

(Manuscript received 9 July 2008, in final form 22 October 2008)

\begin{abstract}
A climatology of the three-dimensional life cycle of the gravest waves in the tropical lower and middle stratosphere is presented. It shows that at periods around 10 days the gravest waves correspond to Kelvin and Rossby-gravity wave packets that substantially affect specific regions in the lower stratosphere. It also shows that the planetary-scale Kelvin waves with zonal wavenumber $s=1$ and periods between 10 and 20 days produce a substantial signal. Still at the planetary scales, the climatology also shows that the global planetary Rossby waves with $s=1$ and periods around 5 and 16 days have a substantial equatorial signature. This climatology is for all the dynamical fields (horizontal wind, temperature, and geopotential height) and relates the equatorial waves to the equatorial zonal mean flow evolution associated with the quasi-biennial oscillation.

The method used to extract the climatology is a composite analysis of the dynamical fields keyed on simple indexes measuring when the waves enter in the stratosphere. For the Kelvin waves, the Rossby-gravity waves, and the 5- and 16-day Rossby planetary waves, these indexes are related to the latitudinal means over the equatorial band of the temperature, the meridional wind, the geopotential height, and the zonal wind respectively. The method is applied first to ERA-40 and then to a simulation done with the LMDz GCM. When compared to the results from ERA-40, this reveals that the LMDz GCM underestimates the Rossbygravity wave packets and a fraction of the Kelvin wave packets. This deficit is attributed to the fact that the model has a too coarse vertical resolution and an insufficient tropospheric forcing for the horizontal wavenumbers $s>3$.
\end{abstract}

\section{Introduction}

The equatorial waves play an important role in the equatorial stratosphere. Equatorial Kelvin waves and Rossby-gravity waves partly produce the quasi-biennial oscillation (QBO) through wave-mean flow interaction (Holton and Lindzen 1972; Baldwin et al. 2001), and the Kelvin waves are considered to be responsible for the westerly phase of the semi-annual oscillation. They can also contribute to the dehydration of the air at the tropical tropopause (Jensen et al. 2001; Fujiwara et al. 2001).

The theory of equatorial waves was developed by Matsuno (1966). The 15-day Kelvin waves and the 4-5day Rossby-gravity waves were first observed in stratospheric soundings by Wallace and Kousky (1968) and Yanai and Maruyama (1966), respectively. Since then,

Corresponding author address: François Lott, Laboratoire de Météorologie Dynamique du CNRS, Ecole Normale Supérieure, 24, Rue Lhomond, 75231 Paris CEDEX 05, France.

E-mail: flott@lmd.ens.fr many studies have documented the presence of equatorial waves in vertical soundings and ground-based observations using radar, lidar, rockets (Tsuda et al. 1994; Sasi et al. 2003; Fujiwara et al. 2003) or ultralong-duration balloons (Vial et al. 2001; Hertzog and Vial 2001). In addition to these equatorially trapped waves, there is a growing amount of evidence that the dynamics in the lower equatorial stratosphere is also modulated by the planetary Rossby waves that were described at higher altitudes and latitudes by Hirota and Hirooka (1984; see also Madden 1978, 2007).

Although the stratospheric equatorial waves are in good part forced by convection (Holton 1972; Manzini and Hamilton 1993), Hendon and Wheeler (2008) have given evidences that they are quite different from the convectively coupled equatorial waves described by Wheeler et al. (2000) that travel coherently with convective centers in the troposphere (Hendon and Wheeler 2008). This is because the coupled modes are rather slow: their periods correspond to small vertical wavelengths in the stratosphere, where they dissipate rapidly. 
With the meteorological satellites, the global observations have yielded a more comprehensive description of the planetary and equatorially trapped waves in the tropical stratosphere (Rodgers 1976; Randel and Gille 1991; Mote et al. 2002; Mote and Dunkerton 2004). Recently, Ern et al. (2008) have analyzed waves with zonal wavenumbers below $s=6-7$ in the temperature profiles from the Soundings of the Atmosphere Using Broadband Emission Radiometry (SABER) instrument. They have also shown that the European Centre for Medium-Range Weather Forecasts (ECMWF) analysis reproduces the Kelvin, and the gravest Rossby waves well; but underestimates the amplitude of the Rossbygravity waves by a factor near 2 . This underestimation was also shown on one particular Rossby-gravity wave in comparison with constant level balloon flights data in Vial et al. (2001).

A large number of studies have analyzed the extent to which the middle atmosphere general circulation models (GCMs) simulate the equatorial waves (Boville and Randel 1992; Manzini and Hamilton 1993). They show that the GCMs can produce equatorial waves but that their amplitudes are strongly sensitive to the convection parameterizations (Horinouchi et al. 2003) and to the vertical resolution (Boville and Randel 1992). It is also difficult to evaluate if those models reproduce the equatorial waves with accuracy, both because the equatorial waves are modulated by the QBO and because the QBO is absent from a good part of the models used in Horinouchi et al. (2003), for instance. Furthermore, even if a model simulates a QBO, it does not necessarily mean that it simulates the resolved waves properly because the momentum fluxes producing the QBO in models are partly due to the gravity wave parameterizations (Giorgetta et al. 2006): those can be tuned to compensate for the model errors on the resolved waves.

To gain further insight into the GCMs' ability to simulate the equatorial waves, the results from SABER in Ern et al. (2008) are instructive: they show that ECMWF analysis can be used to validate the GCMs. It is in this context that Tindall et al. $(2006 \mathrm{a}, \mathrm{b})$ proposed an equatorial wave detection method that they apply to the 15-yr ECMWF Re-Analysis (ERA-15) but that could also be applied to GCMs. Their method mixes spectral analysis techniques with the equatorial wave theory, as was done for the equatorial troposphere by Yang et al. (2003).

The Tindall et al. (2006a,b) and Yang et al. (2003) papers illustrate well how the statistical methods mixing theory and data analysis techniques can be used to extract waves. They also open a debate about the amount of theory that can be introduced into a statistical method before it ceases to be useful. If the data are projected onto well-defined theoretical solutions, or onto too many of them, the results can be biased toward these solutions or correspond to a signal of very small amplitude. Furthermore, if one tries to analyze the data by projecting them onto each horizontal wavenumber, one can miss the fact that Kelvin and Rossby-gravity waves often propagate in the lower stratosphere as wave packets in the horizontal direction [for the Rossby-gravity waves, see Dunkerton and Baldwin (1995)], simply because they are observed not very far above their tropospheric sources. These packets need to be extracted by an appropriate analysis technique if one wishes to test the ability of a GCM to reproduce locally the correct amplitude of the signal due to a given group of waves.

Another issue that arises when validating a GCM against reanalysis data is that of the altitude at which it should be done. Indeed, we cannot disregard the hypothesis that a GCM can simulate well the equatorial waves and not the QBO. In this case, the filtering of the equatorial waves by the mean flow can make the model waves incorrect for wrong reasons. To circumvent this difficulty, we can use the fact that the equatorial waves are forced by convection and enter the stratosphere from below: there is a region in the lower stratosphere where they can be described before they interact with the QBO and because the QBO in the lower stratosphere is in the right phase over a sufficient depth to permit us to identify the waves' vertical propagation.

The method we propose in this paper fully takes into account these points. It is a composite analysis of filtered dynamical fields, keyed to indexes measuring when a particular wave enters the stratosphere. To ensure that we extract signals of substantial amplitudes, the time-space bandpass filters we use have broad bands, and the indexes we choose only retain one general result from the equatorial wave theory. This result is that for the Kelvin waves and the $n=1$ and $n=3$ planetary Rossby waves, the zonal velocity $u$, the temperature $T$, and the geopotential height $Z$ have a uniform sign when the latitude $\phi$ varies over the equatorial band. Here, $n$ is the number of nodes for the meridional velocity between the poles. For the Rossby-gravity waves, the same property applies to the meridional wind $v$. The log-pressure altitude chosen for this index is $z=21 \mathrm{~km}$ : it is well above the tropospheric sources of the waves but still at a rather low level in the stratosphere to allow us to compare datasets with different QBO signals.

Although composite methods are often used in the troposphere to characterize the equatorial waves that modulate the tropical meteorology at the intraseasonal scale (Wheeler et al. 2000) or to characterize the spatiotemporal patterns of the intraseasonal oscillation 
(Matthews 2000), they are not often used to characterize the waves in the equatorial stratosphere. This is a clear deficit because the composite method can extract signals that can have a clear dynamical interpretation and that amplitudes can compare with particular case studies.

The plan of the paper is as follows: Section 2 presents a spectral analysis of dynamical fields averaged over the equatorial band and in the lower stratosphere of the 40-yr ECMWF Re-Analysis (ERA-40; Uppala et al. 2005). It permits to identify the spectral domain over which various waves modulate the equatorial lower stratosphere. Still for ERA-40, section 3 presents composite analyses of the trapped equatorial wave packets and compares the composites to particular cases. Section 4 presents composite analyses of the $s=1$ Kelvin waves and the $s=1$ symmetric planetary Rossby waves that also affect the equatorial stratosphere. Section 5 repeats the analysis for the GCM of the Laboratoire de Météorologie Dynamique (LMDz; Hourdin et al. 2006), emphasizing the differences from the results from ERA-40.

\section{Spectral analysis and composite method}

\section{a. Spectral analysis}

To illustrate that the meridional mean of dynamical quantities can serve as an indicator of the gravest waves affecting the lower and middle stratosphere, we next use daily products from the six pressure levels in ERA-40 that are in the lower stratosphere $(P=100,70,50,30$, 20 , and $10 \mathrm{hPa}$ ). Because most of the data relevant for the tropical stratosphere are from satellites, we will only consider the last $25 \mathrm{yr}$ of the reanalysis (1978-2002). From this dataset we will estimate the space-time spectra of $\langle T\rangle,\langle Z\rangle,\langle u\rangle$, and $\langle v\rangle$ respectively. Here, \langle\rangle indicates the average in latitude over $10^{\circ} \mathrm{S}-10^{\circ} \mathrm{N}$. To evaluate these spectra, we first extract the double Fourier transform of the corresponding fields for each year:

$$
\langle X\rangle(\lambda, z, d, y)=\sum_{s=0}^{s=n_{\mathrm{lo}} / 2-1} \sum_{j=-182}^{j=182}\langle\hat{X}\rangle\left(s, z, \omega_{j}, y\right) e^{i\left(s \lambda-\omega_{j} d\right)},
$$

where $X$ is for $u, v, Z$, or $T$. In Eq. (1), $\lambda$ is the longitude and $z=H \log \left(P_{r} / P\right)$ is the $\log$-pressure coordinate, with $H=7 \mathrm{~km}$ and $P_{r}=1000 \mathrm{hPa}$; also, $d$ is the day ( $d=1365,1$ day being dropped every 4 yr $), y$ the year, $s$ the zonal wavenumber, $n_{\mathrm{lo}}$ the number of longitudes, and $\omega_{j}=2 \pi j / 365$ the frequency. We then evaluate the periodogram, $\langle\hat{X}\rangle\langle\hat{X}\rangle^{*}$, where the star denotes the complex conjugate, and estimate the spectra $S_{\langle X\rangle}(s, \omega)$ by averaging the periodograms over $25 \mathrm{yr}$ and over the six vertical levels. To reduce even further the pointto-point variability in our estimation of the spectra, we also smooth them by a nine-point boxcar average, yielding to a frequency resolution around 0.025 cycles day $^{-1}$. In the following these spectra are shown in a conventional energy-conserving log axis representation, where $s \omega S_{\langle x\rangle}(s, \omega)$ is shown as a function of $\log (s)$ and $\log (\omega)$. This presentation has the advantage of providing a good representation of the broad spectral peaks that characterize wave packet propagation.

The spectrum of $\langle T\rangle\left[S_{\langle T\rangle}(s, \omega)\right]$ in Fig. 1a shows a well-defined local maximum in the eastward direction for $s \approx 2-6$ and for periods $\omega^{-1} \approx 3-10$ days. Note also the presence of a secondary maximum for $S_{\langle T\rangle}(s, \omega)$ at the horizontal wavenumber $s=1$, in the eastward direction, and for periods around $\omega^{-1} \approx 10-20$ days. These maxima are likely to be due to equatorial Kelvin waves (Shiotani et al. 1997). Note that this wave signal is quite distinct from the Madden-Julian oscillation (MJO) signal, which is slower (its periods are larger than 30 days). This shows that if the MJO is associated with Kelvin waves coupled to the convection, these waves are too slow to propagate deeply in the stratosphere without being dissipated. Compared to these two extrema, the spectrum $S_{\langle T\rangle}(s, \omega)$ shows relatively little power in the westward direction.

In the eastward direction, the spectrum of $\langle Z\rangle$, $S_{\langle Z\rangle}(s, \omega)$ in Fig. 1b resembles that of $\langle T\rangle$ in Fig. 1a. In the westward direction, $S_{\langle Z\rangle}(s, \omega)$ shows two extrema for $s=1$ at periods $\omega^{-1} \approx 5$ days and $\omega^{-1} \approx 16$ days respectively. These two periods correspond to those of the planetary Rossby waves (Madden 1978, 2007), which apparently affect substantially the equatorial lower stratosphere. Note that the maximum around $\omega^{-1} \approx 16$ days is quite broad (it covers the periods $\omega^{-1} \approx 10-20$ days), a spreading of the variance from which it follows that the "slow" normal modes are affected by the seasonal changes in the midlatitudes zonal winds (Salby 1981).

The spectrum of $\langle u\rangle-S_{\langle u\rangle}(s, \omega)$ in Fig. 1c-also shows significant power in both directions. In the eastward direction, the shape of $S_{\langle u\rangle}$ again resembles that of $\langle T\rangle$ in Fig. 1a. In the westward direction, $S_{\langle u\rangle}(s, \omega)$ presents two maxima for $s=1$, corresponding to the two maxima in the spectra for $\langle Z\rangle$ in Fig. $1 b$.

Finally, the spectrum of $\langle v\rangle-S_{\langle v\rangle}(s, \omega)$ in Fig. 1d-is dominated by a maximum in the westward direction for periods $\omega^{-1} \approx 2-10$ days and for zonal wavenumbers $s \approx 3-9$; this is likely to be due to the Rossby-gravity waves (Ern et al. 2008).

\section{b. Composite method}

To extract the waves we next apply bandpass filters to all the fields. These filters are designed in the spectral 

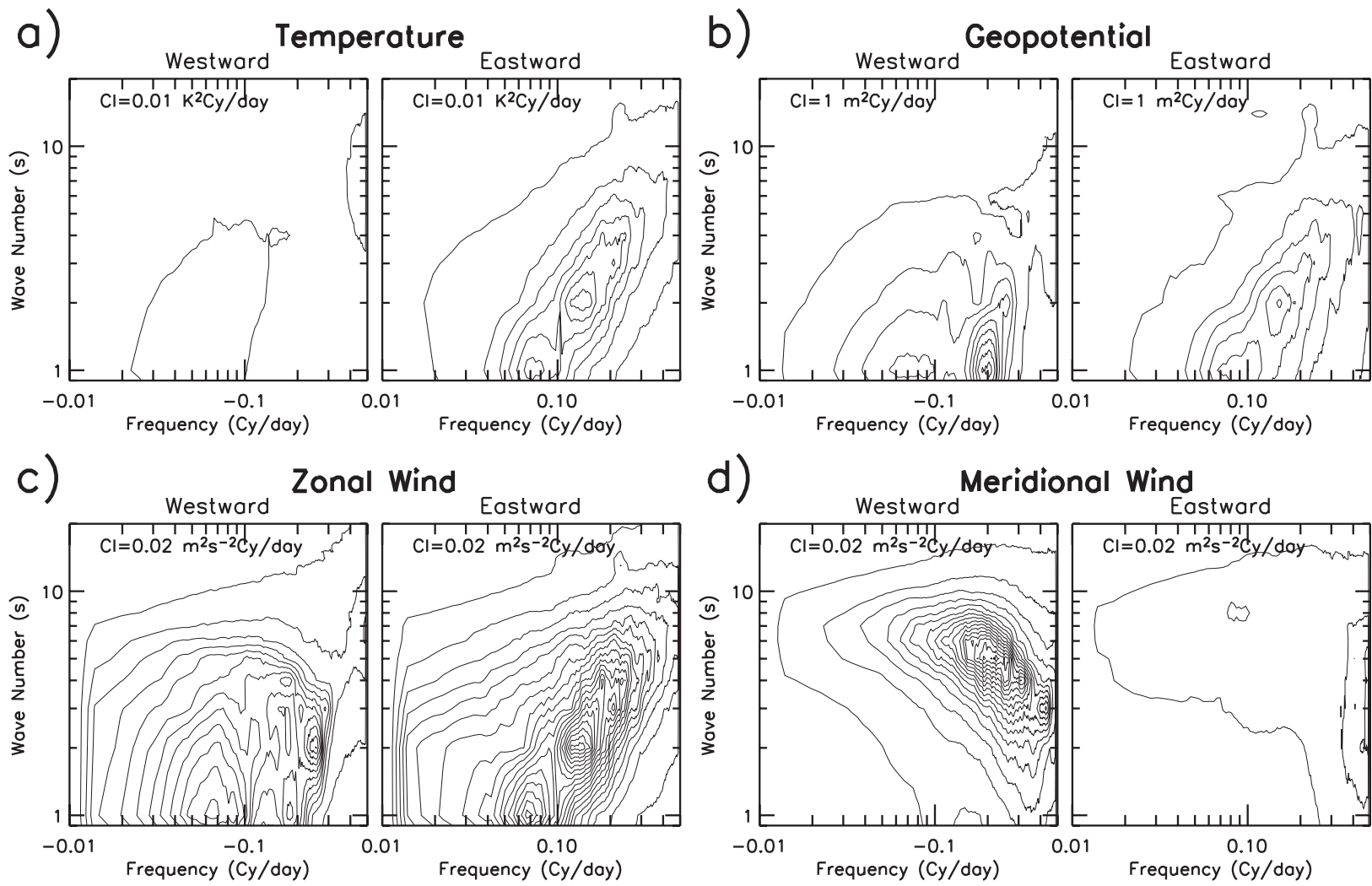

FIG. 1. ERA-40: Space-time spectra of fields averaged between $10^{\circ} \mathrm{S}$ and $10^{\circ} \mathrm{N}$ in the lower and middle stratosphere: (a) temperature, (b) geopotential height, (c) zonal wind, and (d) meridional wind. In each plot are presented $s \omega S_{\langle x\rangle}(s, \omega)$ to accommodate the log-scale of the two axes.

space by using transfer functions, the spectra of which largely contain the various maxima identified in Fig. 1. More specifically, for each altitude $z$ and latitude $\phi$, we first evaluate the time-longitude double Fourier transform of the fields and multiply the Fourier coefficients by the Fourier transform of the transfer function. For a given dynamical field $X$, the filtered value $\tilde{X}$ is given by

$$
\tilde{X}(\lambda, \phi, z, t)=\sum_{s=0}^{n_{\mathrm{lo}} / 2} \sum_{j=-J / 2+1}^{J / 2-1} \hat{f}\left(\omega_{j}\right) \hat{g}(s) \hat{X}\left(s, \phi, z, \omega_{j}\right) e^{i\left(s \lambda-\omega_{j} t\right)},
$$

where the discrete periods $\omega_{j}=j(2 \pi / J)$, with $J=9130$ days (the number of days in the 25 -yr long dataset). In all cases, the Fourier transform of the time filter $f(t)$ is of the form

$$
\hat{f}(\omega)=\left\{\begin{array}{ccc}
0 & \text { for } \omega \leq f_{1} \text { and for } f_{4} \leq \omega \\
\left\{1-\cos \left[\pi\left(\omega-f_{1}\right) /\left(f_{2}-f_{1}\right)\right]\right\} / 2 & \text { for } & f_{1} \leq \omega \leq f_{2} \\
1 & \text { for } & f_{2} \leq \omega \leq f_{3} \\
\left\{1-\cos \left[\pi\left(\omega-f_{4}\right) /\left(f_{3}-f_{4}\right)\right]\right\} / 2 & \text { for } & f_{3} \leq \omega \leq f_{4},
\end{array}\right.
$$

while the Fourier transform of the horizontal filter $g(\lambda)$ is of the form

$$
\begin{aligned}
\hat{g}(s) & =H\left(s-s_{1}\right) H\left(s_{2}-s\right), \quad \text { where } \quad H(s<0)=0, \\
H(s=0) & =0.5, \quad \text { and } H(s>0)=1 .
\end{aligned}
$$

Table 1 gives the values for $f_{i}$ and $s_{i}$ we have used to extract the different waves.

To build the composites, we first define an index $A_{\langle\tilde{X}\rangle}(t)$, which is the maximum value of the filtered field $\langle\tilde{X}\rangle$ $(\lambda, z=21 \mathrm{~km}, t)$ when the longitude $\lambda$ varies and at the 
TABLE 1. Coefficient of the various filters in Eqs. (3) and (4).

\begin{tabular}{ccccccc}
\hline \hline & \multicolumn{3}{c}{ Equatorially trapped waves } & & \multicolumn{2}{c}{ Planetary waves } \\
\cline { 2 - 3 } \cline { 6 - 7 } & Kelvin & Kelvin & Rossy-gravity & & 5 -day & 16 -day \\
& $s=1$ & $s=2-5$ & $s=4-8$ & & $s=1$ & $s=1$ \\
\hline$f_{1}^{-1}$ & 40 & 13 & -1 & & -1 & -5 \\
$f_{2}^{-1}$ & 25 & 10 & -3 & & -3 & -10 \\
$f_{3}^{-1}$ & 10 & 3 & -8 & & -8 & -25 \\
$f_{4}^{-1}$ & 5 & 1 & -12 & & -12 & -40 \\
$s_{1}$ & 0.5 & 1 & 3 & & 0.5 & 0.5 \\
$s_{2}$ & 1.5 & 6 & 9 & & 1.5 & 1.5 \\
$X$ & $T$ & $T$ & $v$ & $Z$ & $u$ \\
\hline
\end{tabular}

key altitude $z=21 \mathrm{~km}$. Each time, we also identify the longitude $\lambda_{M}(t)$ at which this maximum in $\langle\tilde{X}\rangle$ occurs:

$$
A_{\langle\tilde{X}\rangle}(t)=\langle\tilde{X}\rangle\left(\lambda_{M}, z=21 \mathrm{~km}, t\right) .
$$

Then the composites are built from maps of $\tilde{u}, \tilde{v}, \tilde{T}$, and $\tilde{Z}$ and of the zonal mean zonal wind $\bar{u}$ selected when $A_{\langle\tilde{X}\rangle}$ presents a local maximum that exceeds in amplitude a given threshold. In all the cases analyzed in this paper, this threshold is such that exactly $N_{c}=25$ events are selected: that is, around one event per year is used. Note also that for all these events, the maps entering in the composite are phase locked one with the others, by applying to each of them a constant horizontal shift in longitude equal to $-\lambda_{M}$ : it is $\tilde{X}(\lambda-$ $\left.\lambda_{M}, \phi, z, t\right)$ that enters in the composite rather than $\tilde{X}(\lambda, \phi, z, t)$. Finally, note that at a given nonzero lag $l \neq$ 0 the composites are built with those maps that correspond to a date situated at the given lag $l$ from a local maximum in $A_{\langle\tilde{X}\rangle}$ identified before. In all the cases presented, we take $-L<l<L=20$ days, where $L$ is a time window parameter. In the rest of the paper we will denote the composites by the superscript $\mathcal{C}$, and the composite of the 4D filtered fields will be written

$$
\tilde{X}^{\mathcal{C}}(\lambda, \phi, z, l) \text {. }
$$

\section{Equatorial wave packets}

\section{a. Kelvin waves}

To extract the oscillations that produce the broad $s \approx 2-6$ and $\omega^{-1} \approx 3-10$ days maximum in the eastward direction in Figs. 1a-c, we take for the filter temporal parameters in Eq. (3) $f_{1}^{-1}=17$ days, $f_{2}^{-1}=14$ days, $f_{3}^{-1}=$ 3 days, and $f_{4}^{-1}=1$ day and, for the spatial parameters in Eq. (4), $s_{1}=1$ and $s_{2}=6$ (see also the half-power point of the filter in Fig. 2 indicated by "Kelvin $s=2-5$ "). We then take for $X$ in the wave index definition in Eq. (5) the filtered value of the temperature at $z=21 \mathrm{~km}$.

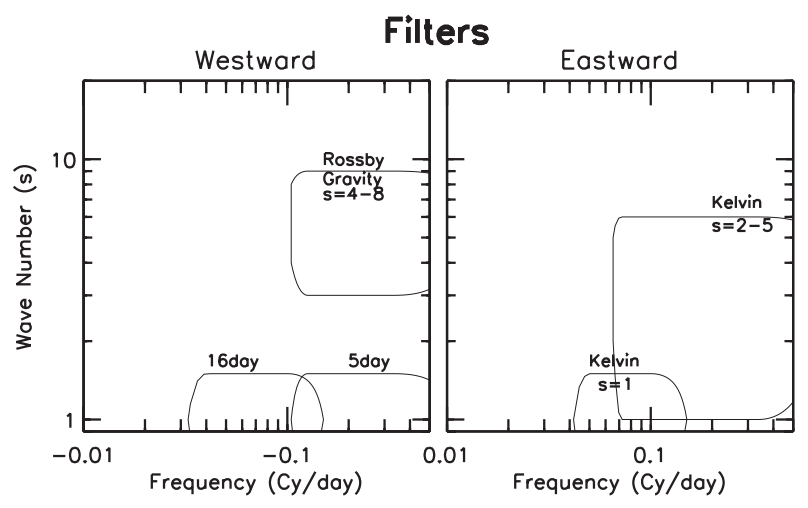

FIG. 2. Half-power points of the space-time filters used to extract the gravest waves in the equatorial lower stratosphere.

Our choice here to group together the wavenumbers $s=2$ to $s=5$ (rather than another subset including, for instance, $s=1$ ) is somehow arbitrary. It is motivated by the observational fact that in the stratosphere the Kelvin waves often propagate as packets dominated by the wavenumbers $s \geq 2$. To illustrate this point, the thin black line in Fig. 3a shows the temporal evolution of the Kelvin wave index $A_{\langle\tilde{T}\rangle}$. It shows that the index is very large, for instance near the beginning of 1999. If we look at the evolution of the unfiltered temperature at the equator near this peak in $A_{\langle\tilde{T}\rangle}$, we see in Fig. $3 \mathrm{~b}$ an eastward-propagating anomaly, starting around 9 February, at longitude $\lambda \approx 30^{\circ} \mathrm{E}$ and ending almost 10 days later at $\lambda \approx 140^{\circ} \mathrm{E}$. Its phase speed is around $C \approx 25 \mathrm{~m} \mathrm{~s}^{-1}$. In Fig. $3 \mathrm{a}$ we also see that the index is larger when the equatorial zonal mean zonal wind $(\bar{u}$, where the overbar is for the zonal average) at $z=21 \mathrm{~km}$ is negative (thick gray line in Fig. 3a), which is consistent with the picture that waves with positive phase velocity develop more easily when $\bar{u}<0$ and because larger intrinsic phase speeds yield to larger vertical wavelengths [this was also found by Randel and Wu (2005) and Ern et al. (2008)].

The composites of the geopotential height $\tilde{Z}^{c}$ and of the horizontal winds $\left(\tilde{u}^{c}, \tilde{v}^{c}\right)$, at $l=0$ day lag are shown in Fig. 4a. They have the characteristic structure of a Kelvin wave packet, where the maximum values for the horizontal wind and for the geopotential height are around $3 \mathrm{~m} \mathrm{~s}^{-1}$ and $14 \mathrm{~m}$, respectively. These values are substantial when compared to the standard deviations for the corresponding unfiltered quantities (first row in Table 2); and they are also significant at the 1\% level (second column in Table 2), and using a normal distribution test for the sum of $N_{c}=25$ independent realizations which standard deviation equals that of the unfiltered signal ( $\sigma$ in the first column of Table 2).

The Hovmöller diagram presenting the composite evolution of the temperature $\left\langle\tilde{T}^{c}\right\rangle$ is in Fig. 4b. It shows 
a)
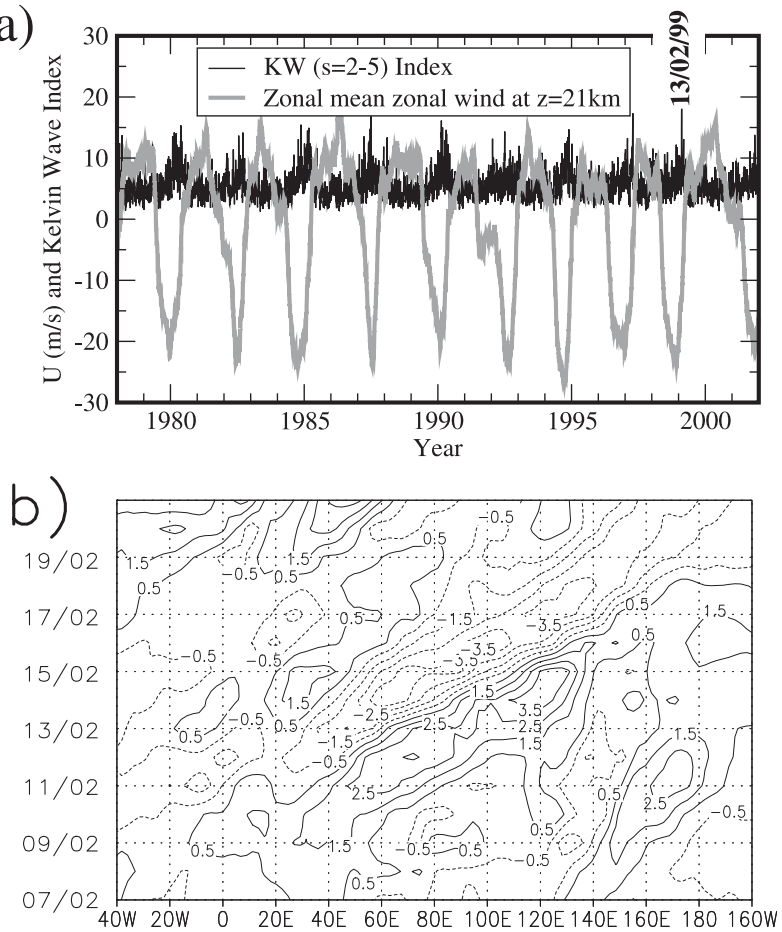

FIG. 3. ERA-40: Index for the Kelvin wave packets with $s>1$ at $z=21 \mathrm{~km}$. (a) Index $A_{\langle\tilde{u}\rangle}$ in Eq. (5) for the filter parameters in the second row of Table 1 (thin solid), zonal mean zonal wind at the equator and at $z=21 \mathrm{~km}$ (thick gray). (b) Hovmöller diagram of the $z=21 \mathrm{~km}$ temperature anomaly at the equator $(T-\bar{T})$ and for a selected 15-day period in February 1999. The contour interval (CI) is $1 \mathrm{~K}$; negative values are dashed.

eastward propagation as expected, the period is around $\omega^{-1} \approx 7$ days, and the phase speed is around $C \approx 25$ $\mathrm{m} \mathrm{s}^{-1}$. Note also that this composite moves eastward in amplitude: most of the signal at negative lag is to the west of the reference longitude 0 whereas most of the signal at positive lag is to the east. This indicates that the group velocity is eastward, as expected for Kelvin waves. Finally, note that the composite in Fig. $4 \mathrm{~b}$ captures all the characteristics of the particular case shown in Fig. $3 b$.

Figure $4 \mathrm{c}$ is a longitude-altitude diagram of the composite geopotential height at $l=0$ day lag. It is tilted eastward, indicating vertical propagation, and the vertical wavelength is around $\lambda_{z} \approx 10 \mathrm{~km}$. This is a little larger than the theoretical values of $\lambda_{z}$ predicted by the equatorial waves theory when $s \geq 2$ and $\omega^{-1}=7$ days (the theory predicts $\lambda_{z}=8.9 \mathrm{~km}$ when $s=3$ ). Because we find that most of the events selected correspond to periods when $\bar{u}<0$ in the lower stratosphere, this discrepancy is in good part due to the fact that the waves intrinsic frequency are larger than their absolute frequency.

Figure $4 \mathrm{~d}$ is a time-altitude diagram of the Kelvin waves amplitude measured by

$$
\sqrt{\overline{\left\langle\tilde{u}^{c}\right\rangle^{2}}(l, z)}
$$

where the overbar is for the zonal average. It is positively tilted (as expected for upward propagation) and shows that the complete life cycle of the Kelvin waves lasts around 20 days. Above $z=21 \mathrm{~km}$, the wave amplitude is almost constant and then decays above $z=26 \mathrm{~km}$. Because nondissipated disturbances exponentially grow with altitude to equilibrate the density decay, the absence of such an exponential growth above $z=21 \mathrm{~km}$ indicates their attenuation.

Another indication that these waves are dissipated is that they seem to interact with the mean flow. This is indicated by the solid and dashed lines in Fig. 4d, which represent the composite of the zonal mean zonal wind tendency:

$$
\left\langle\bar{u}^{c}\right\rangle(z, l)-\frac{1}{2 L+1} \sum_{l=-L}^{+L}\left\langle\bar{u}^{c}\right\rangle(z, l) .
$$

It shows that the zonal mean zonal wind increases during the characteristic life cycle of the Kelvin waves, in agreement with the picture that Kelvin waves accelerate the zonal mean flow when they are dissipated. Note, however, that for the events selected, the zonal mean zonal wind is quite systematically negative at $s=21 \mathrm{~km}$ (Fig. 3a). In these situations the QBO signal shifts to positive phase aloft, so a good part of the acceleration seen in Fig. 4d results from the fact that we sample dates when the QBO increases in the lower stratosphere.

To corroborate that some dissipations nevertheless occur, we have analyzed for each event the zonal mean zonal wind in the lower stratosphere. In $50 \%$ of the events, the zonal mean zonal wind is above $15 \mathrm{~m} \mathrm{~s}^{-1}$ somewhere between $21 \mathrm{~km}<z<32 \mathrm{~km}$; and because our Kelvin waves have phase velocities around $C \approx$ $15-25 \mathrm{~m} \mathrm{~s}^{-1}$, they sometimes approach a critical level.

\section{b. Rossby-gravity waves}

To extract the oscillations that produce the broad maximum for $\omega^{-1} \approx 2-10$ days and $s \approx 3-9$ in the westward direction of the spectra $S_{\langle v\rangle}$ (Fig. 1d), we take for the filter parameters in Eqs. (3) and (4) $f_{1}^{-1}=-1$ days, $f_{2}^{-1}=-3$ days, $f_{3}^{-1}=-8$ days, $f_{4}^{-1}=-12$ days, $s_{1}=3$, and $s_{2}=9$ (see the curve labeled "Rossby-gravity $s=$ 4-8" in Fig. 2). We take for $X$ in the index definition in Eq. (5) the meridional wind $v$.

The thin black line in Fig. 5a shows the temporal evolution of the wave index $A_{\langle\tilde{v}\rangle}$, while the thick gray line shows the evolution of the equatorial zonal mean zonal wind $\bar{u}$ at $z=21 \mathrm{~km}$. It shows that the index is larger when $\bar{u}>0$, from which it follows that waves with 

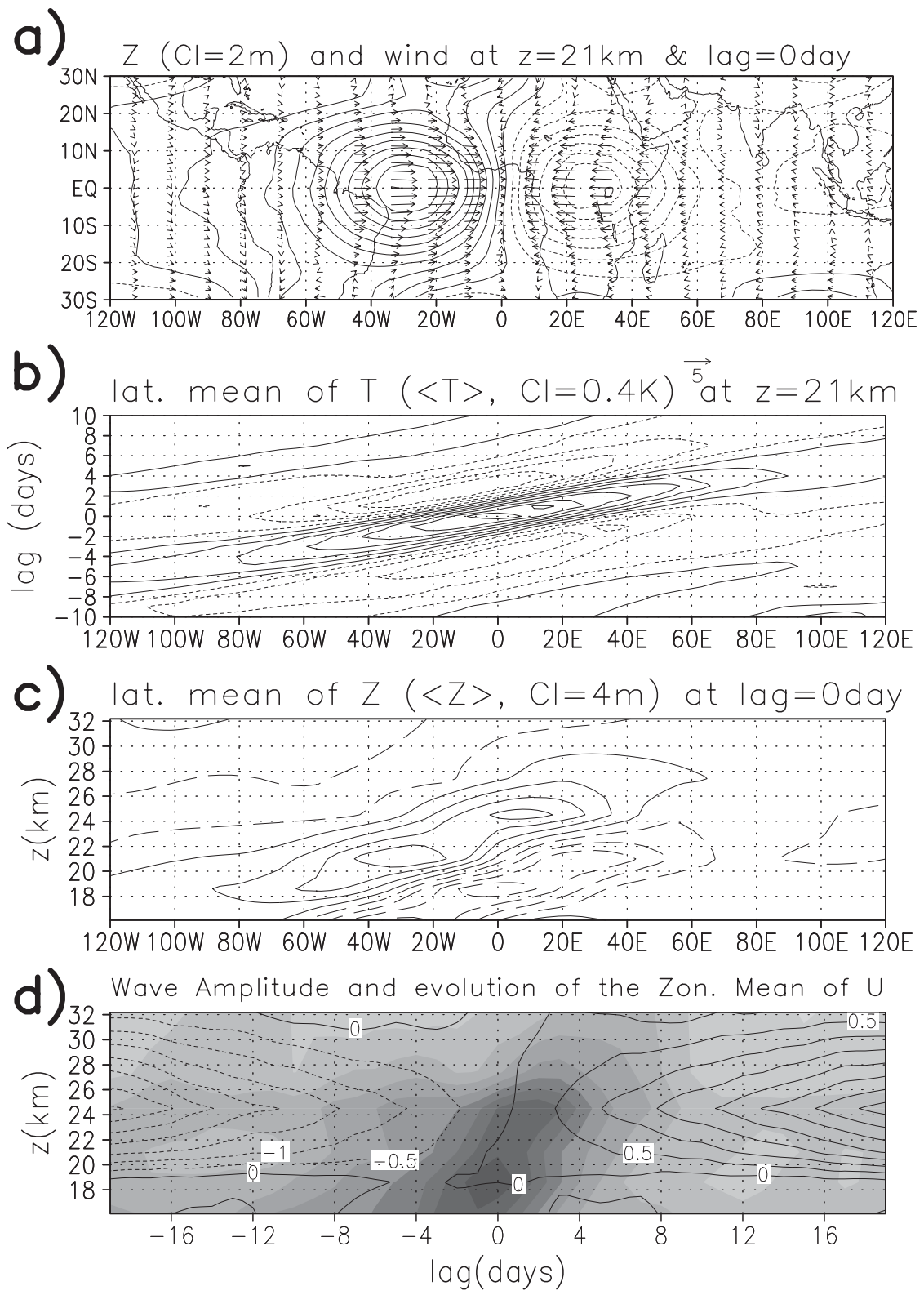

FIG. 4. ERA-40: Composite of the Kelvin waves with wavenumbers $s=2-5$, and periods $\omega^{-1} \approx 3-10$ days (phase speed $C \approx 25 \mathrm{~m} \mathrm{~s}^{-1}$ ). (a) Geopotential and horizontal wind $\left(\tilde{Z}^{c}, \tilde{u}^{c}\right.$, and $\left.\tilde{v}^{c}\right)$ at $z=21 \mathrm{~km}$ and $l=0$ day lag (the geography is arbitrary). (b) Hovmöller diagram of the temperature averaged over the equatorial band $\left\langle\tilde{T}^{c}\right\rangle$ at $z=21 \mathrm{~km}$. (c) Geopotential averaged over the equatorial band $\left\langle\tilde{Z}^{c}\right\rangle$ at $l=0$ day lag. (d) Wave amplitude measured by $\sqrt{\left\langle\tilde{u}^{c}\right\rangle^{2}}$. CI $=0.2 \mathrm{~m} \mathrm{~s}^{-1}$; contours indicate zonal mean zonal wind evolution [see Eq. (8)].

negative phase velocity develop more easily when the zonal mean zonal wind is positive. The wave index $A_{\langle\tilde{v}\rangle}$ also present a pronounced maxima near the end of 1995 (the exact date is 25 September 1995). The evolution of the unfiltered meridional wind at the equator near this peak in $A_{\langle\tilde{v}\rangle}$ is shown in Fig. 5b and presents well-defined westward-propagating anomalies where maxima and minima drift eastward at a phase velocity
$C \approx-15 \mathrm{~m} \mathrm{~s}^{-1}$. The complete duration of this oscillation is around 10 days, and its period at a given longitude is around 5-6 days.

Figure 6a shows the composites of the geopotential height and of the horizontal wind at $l=0$ day lag and at $z=21 \mathrm{~km}$. The pattern is that of a Rossby-gravity wave with westward phase velocity (Matsuno 1966). Note also that the amplitude of the composite in Fig. 6a is 
TABLE 2. Variance statistics of the disturbance fields $u, v, T$, and $Z$ and in the low and middle equatorial stratosphere $(16 \mathrm{~km} \leq z \leq$ $\left.32 \mathrm{~km},-10^{\circ} \mathrm{N} \leq \phi \leq+10^{\circ} \mathrm{N}\right)$, with the zonal means and annual cycle subtracted. First column is std $\operatorname{dev} \sigma$. Second column is $1 \%$ confidence level from a normal distribution test for the sum of $N_{c}=25$ realizations of a random process with std $\operatorname{dev} \sigma$.

\begin{tabular}{lll}
\hline \hline & \multicolumn{1}{c}{$\sigma$} & $1 \%$ for 25 cases \\
\hline$u$ & $4.4 \mathrm{~m} \mathrm{~s}^{-1}$ & $2.2 \mathrm{~m} \mathrm{~s}^{-1}$ \\
$v$ & $2.2 \mathrm{~m} \mathrm{~s}^{-1}$ & $1.1 \mathrm{~m} \mathrm{~s}^{-1}$ \\
$T$ & $0.8 \mathrm{~K}$ & $0.4 \mathrm{~K}$ \\
$Z$ & $12 \mathrm{~m}$ & $6 \mathrm{~m}$ \\
\hline
\end{tabular}

significant for all quantities when compared to the total variance in each fields (see Table 2). Finally note also that in the lower stratosphere, Fig. 6a shows that a characteristic Rossby-gravity wave packet does not extend horizontally over more than $80^{\circ}$ in longitude, which corresponds to two to three wave crests, as in the particular case in Fig. 5b.

Figure $6 \mathrm{~b}$ is a Hovmöller diagram for $\left\langle\tilde{v}^{c}\right\rangle(\lambda, z=21$ $\mathrm{km}, l)$ and shows westward phase propagation with period around 5-6 days. It also shows that the wave packets shift in amplitude toward the east, which indicates eastward group velocity, as expected for Rossby-gravity waves. Finally, it is again important to emphasize that Fig. 6b closely resembles the particular case in Fig. 5b.

Figure $6 \mathrm{c}$ shows a longitude-altitude section of $\left\langle\tilde{v}^{c}\right\rangle(\lambda$, $z, l)$ at $l=0$ day lag. It is tilted eastward with altitude, indicating that the wave packet propagates upward. The vertical wavelength is around $\lambda_{z} \approx 10-12 \mathrm{~km}$. This value falls in the range predicted by the equatorial wave theory (the theory predicts $\lambda_{z}=6 \mathrm{~km}$ for $\omega^{-1} \approx 5$ and $s=5$, and $\lambda_{z}=18 \mathrm{~km}$ for $\omega^{-1} \approx 4 \mathrm{~d}$ and $s=6$ ).

The shaded contours in Fig. 6d represent the time evolution of the composite amplitude measured by

$$
\sqrt{\overline{\left\langle\tilde{\boldsymbol{v}}^{c}\right\rangle^{2}}}(z, l)
$$

The positively tilted structure around $l=0$ day lag indicates upward propagation, and we can see that a complete life cycle duration for the Rossby-gravity waves packet is around 10-15 days. Finally, note as well that the composite amplitude is always quite small above $z=25 \mathrm{~km}$, suggesting that the Rossby-gravity waves contributing to the composite are dissipated above this altitude.

A first indication that this is the case is given by the zonal mean zonal wind evolution during the life cycle of our composite [see Eq. (8) and the black lines in Fig. $6 \mathrm{~d}]$. It shows that the zonal mean zonal wind decreases during the life cycle of the Rossby-gravity waves, in agreement with the fact that these waves decelerate the flow when they are dissipated. Again this is somehow
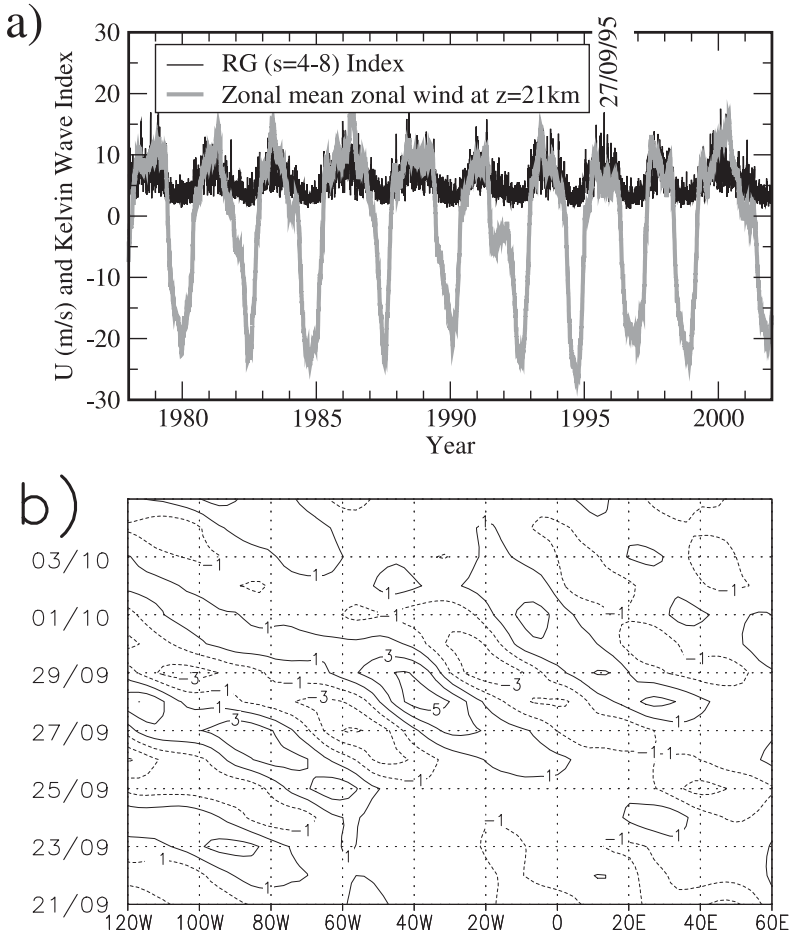

FIG. 5. ERA-40: Index for the Rossby-gravity waves packets with $s>1$ at $z=21 \mathrm{~km}$. (a) Index $A_{\langle\tilde{v}\rangle}$ in Eq. (5) for the filter parameters in the third row of Table 1 (thin solid), zonal mean zonal wind at the equator and at $z=21 \mathrm{~km}$ (thick gray). (b) Hovmöller diagram of the $z=21 \mathrm{~km}$ meridional velocity anomaly at the equator $(v-\bar{v})$ and for a selected 15-day period in September-October 1995. CI is $2 \mathrm{~m} \mathrm{~s}^{-1}$; negative values are dashed.

misleading because we select dates for which the zonal wind is positive at $z=21 \mathrm{~km}$ (see Fig. 5a): in these situations the QBO often moves to its negative phase aloft.

To gain further insights in the physical processes that produce the attenuation of the Rossby-gravity waves in Fig. 6d, we have also analyzed the zonal mean zonal wind at the equator $\bar{u}(\phi=0)$ and for each of the events selected to build the composites in Fig. 6 . We found that for more than $50 \%$ of the events selected, $\bar{u}(\phi=0)$ is less than $-20 \mathrm{~m} \mathrm{~s}^{-1}$ somewhere between 25 and $32 \mathrm{~km}$. Because the Rossby-gravity waves have horizontal phase velocities around $C=-15 \mathrm{~m} \mathrm{~s}^{-1}$, it means that for a large number of the events selected, the Rossby-gravity waves encounter a critical level above $z=25 \mathrm{~km}$.

\section{Kelvin $(s=1)$ and free planetary waves}

Our choice to describe the waves in terms of packets in the horizontal direction is not adapted for some of them. This appears clearly in the spectra of $\langle Z\rangle$ and $\langle u\rangle$ in Figs. 1b,c: they both present two relative maxima in the westward direction for the planetary wavenumber $s=1$ (one for $\omega^{-1} \approx 5$ days and the other for $\omega^{-1} \approx 16$ 

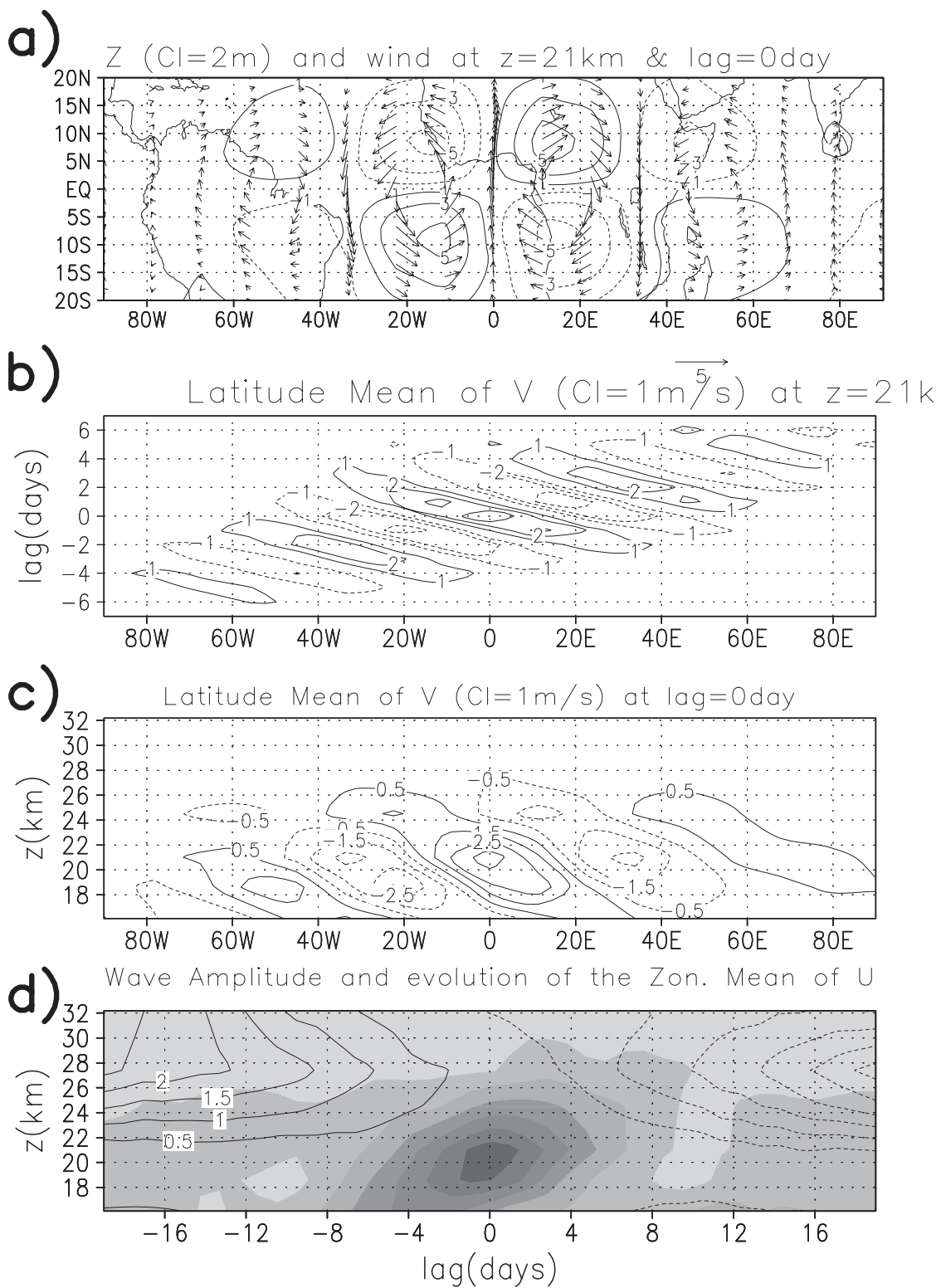

FIG. 6. ERA-40: Composite of the Rossby-gravity waves with wavenumbers $s=4-8$, and periods $\omega^{-1} \approx 3-8$ days (phase speed $C \approx-15 \mathrm{~m} \mathrm{~s}^{-1}$ ). (a) Geopotential and horizontal wind $\left(\tilde{Z}^{c}, \tilde{u}^{c}\right.$, and $\left.\tilde{v}^{c}\right)$ at $z=21 \mathrm{~km}$ and at $l=0$ day lag; the geography is arbitrary. (b) Meridional wind averaged over the equatorial band $\left\langle\tilde{v}^{c}\right\rangle$ at $z=21 \mathrm{~km}$. (c) Meridional wind averaged over the equatorial band $\left\langle\tilde{v}^{c}\right\rangle$ and at $l=0$ day lag. (d) Shaded wave amplitude measured by $\sqrt{\left\langle\tilde{v}^{c}\right\rangle^{2}}$. CI $=$ $0.2 \mathrm{~m} \mathrm{~s}^{-1}$; contours indicate zonal mean zonal wind evolution [see text near Eq. (8)].

days), which are probably due to the planetary Rossby waves. The spectra of $\langle u\rangle$ in Fig. 1c also present a secondary maximum for $s=2$ and $\omega^{-1} \approx 4$ days, which probably has the same origin. Because these are free modes of oscillations, it is evident that they are better described as individual harmonics.

More problematic is the relative maximum for $s=1$ and $\omega^{-1} \approx 10-20$ days, present in the spectra of $\langle T\rangle$, $\langle Z\rangle$, and $\langle u\rangle$ in the eastward direction (Figs. 1a-c). Be- cause this maximum can be due to Kelvin waves, and because it looks quite separated from the broad maximum with $s>1$ we attributed to Kelvin wave packets in section 3, we have to consider that at least a part of the $s=1$ Kelvin waves should be described in terms of individual harmonics. On the one hand, this complementary approach is justified by the fact that there are periods of times when the $s=1$ signal is strongly dominant (not shown, but this is the case at the beginning of 
March 1985) and needs to be analyzed alone. On the other hand, there are also periods of times when an $s=1$ planetary Kelvin wave accompanies the Kelvin wave packets. In this last situation, the resulting composites, including the $s=1$ contribution, are almost like those in section 3a. For this reason, and also because we have excluded a good part of the $s=1$ signal in section $3 \mathrm{a}$, we have to treat the $s=1$ Kelvin waves here for completeness.

\section{a. Kelvin waves}

To capture the $s=1$ eastward-propagating disturbances with periods of 10 days $<\omega^{-1}<20$ days, we take for the filter parameters in Eqs. (3) and (4) $f_{1}^{-1}=40$ days, $f_{2}^{-1}=25$ days, $f_{3}^{-1}=10$ days, $f_{4}^{-1}=5$ days, $s_{1}=$ 0.5 , and $s_{2}=1.5$ (see also Fig. 2). We take for $X$ in the index definition [Eq. (5)] the temperature $T$.

Figure 7 a shows the composites $\tilde{Z}^{c}, \tilde{u}^{c}$, and $\tilde{v}^{c}$ at $l=0$ days lag and $z=21 \mathrm{~km}$. It is a Kelvin wave pattern, in which the geopotential height and the zonal wind amplitudes are around $14 \mathrm{~m}$ and $3 \mathrm{~m} \mathrm{~s}^{-1}$, respectively. These values are again substantial and significant according to Table 2. Figure $7 \mathrm{~b}$ shows a Hovmöller diagram for the evolution of the temperature at $z=21 \mathrm{~km}$ $\left\langle\tilde{T}^{c}\right\rangle(l, \lambda, z=21 \mathrm{~km})$. We see that the composite propagates in the eastward direction, with a period $\omega^{-1} \approx$ 15 days and a phase speed $C \approx 30 \mathrm{~m} \mathrm{~s}^{-1}$. The maximum amplitude for $\left\langle\tilde{T}^{c}\right\rangle$ is about $1.4 \mathrm{~K}$. Figure $7 \mathrm{c}$ shows a vertical-zonal section of the composite geopotential at $l=0$ day lag; that is, $\left\langle\tilde{Z}^{c}\right\rangle(\lambda, z, l=0$ days $)$. It is tilted in the westward direction indicating upward propagation. The vertical wavelength is around $\lambda_{z} \approx 12 \mathrm{~km}$ and is quite close to that predicted by the equatorial wave theory $\left(\lambda_{z}=9.8 \mathrm{~km}\right.$ for $s=1$ and $\omega^{-1}=15$ days).

The temporal evolution of the composite amplitude [see Eq. (7)] in Fig. 7d indicates that the life cycle of these waves lasts around 20-30 days, and the maximum amplitude decays with altitude. This again suggests that the $s=1$ Kelvin waves entering in the composite are dissipated in the lower stratosphere. As for the Kelvin wave packets in section $3 \mathrm{a}$, the life cycle of the composite is accompanied by an increase of the zonal mean zonal wind [see Eq. (8) and the contours in Fig. 7d].

\section{b. Five-day wave}

To capture the oscillations that produce the $s=1$, $\omega^{-1} \approx 5$-day extrema in the westward direction in Figs. $1 \mathrm{~b}, \mathrm{c}$, we take for the filter parameters in Eqs. (3) and (4) $f_{1}^{-1}=-1$ day, $f_{2}^{-1}=-3$ days, $f_{3}^{-1}=-8$ days, $f_{4}^{-1}=$ -12 days, $s_{1}=0.5$, and $s_{2}=1.5$ (see Fig. 2), and we use the geopotential height $Z$ for $X$ in Eq. (5).

The composites $\tilde{Z}^{c}, \tilde{u}^{c}$, and $\tilde{v}^{c}$ at $z=21 \mathrm{~km}$ and $l=0$ day lag in Fig. 8a strongly resemble an $n=1$ Rossby planetary wave. In the tropical regions, the characteristic amplitude of the winds and geopotential height are around $1-2 \mathrm{~m} \mathrm{~s}^{-1}$ and $12 \mathrm{~m}$, respectively. These values are substantial and significant according to Table 2 . It is also clear from Fig. 8a that the disturbances are of global character, the extrema for the geopotential being near the latitudes $40^{\circ} \mathrm{S}$ and $40^{\circ} \mathrm{N}$, in good agreement with the theory of the planetary Rossby waves when $n=1$.

The time-altitude diagram for the geopotential height composite $\left\langle\tilde{Z}^{c}\right\rangle$ in Fig. 8b indicates that the planetary Rossby waves propagate in the westward direction with periods around $\omega^{-1} \approx 5-6$ days, which is close to the period of the $s=1$ planetary Rossby waves observed at $z \approx 50 \mathrm{~km}$ by Hirota and Hirooka (1984). Consistently, the longitude-latitude section of $\left\langle\tilde{Z}^{c}\right\rangle$ at $l=0$ day lag in Fig. $8 \mathrm{c}$ is almost barotropic. Because these Rossby waves are free modes of oscillation, their amplitude in the vertical should vary exponentially as $e^{2 z / 7 H}$, and this is almost the variation of amplitude with altitude we see in Fig. 8c.

To corroborate this point, Fig. $8 \mathrm{~d}$ shows a timealtitude diagram of the composite amplitude weighted by $e^{-2 z / 7 H}$ :

$$
\sqrt{\overline{\left\langle\tilde{Z}^{c}\right\rangle^{2}}} e^{-2 z / 7 H}
$$

It shows that this quantity is almost uniform in altitude at a given time and that the life cycle of these Rossby waves lasts around $7-10$ days.

\section{c. Sixteen-day wave}

To capture the oscillations that produce the $s=1$, $\omega^{-1} \approx 10-25$-day maximum in the westward direction in Figs. 1b,c, we take for the filter parameters in Eqs. (3) and (4) $f_{1}^{-1}=-5$ day, $f_{2}^{-1}=-10$ day, $f_{3}^{-1}=-25$ day, $f_{4}^{-1}=-40$ day, $s_{1}=0.5$, and $s_{2}=1.5$ (see Fig. 2), and we use for $X$ in the wave index definition in Eq. (5) the zonal wind $u$.

The composites $\tilde{Z}^{c}, \tilde{u}^{c}$, and $\tilde{v}^{c}$ at $z=21 \mathrm{~km}$ and $l=0$ day lag in Fig. 9a strongly resemble an $n=3$ planetary Rossby wave (see Madden 1978). The maxima for the geopotential altitude in Fig. 9a are around the latitudes $\phi= \pm 20^{\circ}$, and at a given longitude $\phi, \tilde{Z}^{c}$ changes sign at the latitudes $\phi= \pm 30^{\circ}$. In the tropics, the amplitudes of the winds and of the geopotential altitude are around $3 \mathrm{~m} \mathrm{~s}^{-1}$ and $12 \mathrm{~m}$, respectively. These values are substantial and significant according to Table 2. Note also that the composite of the geopotential becomes larger in the mid and high latitudes than it is in the tropics. These results are consistent with the planetary wave theory, which predicts that for an $n=3$ free planetary wave, the 

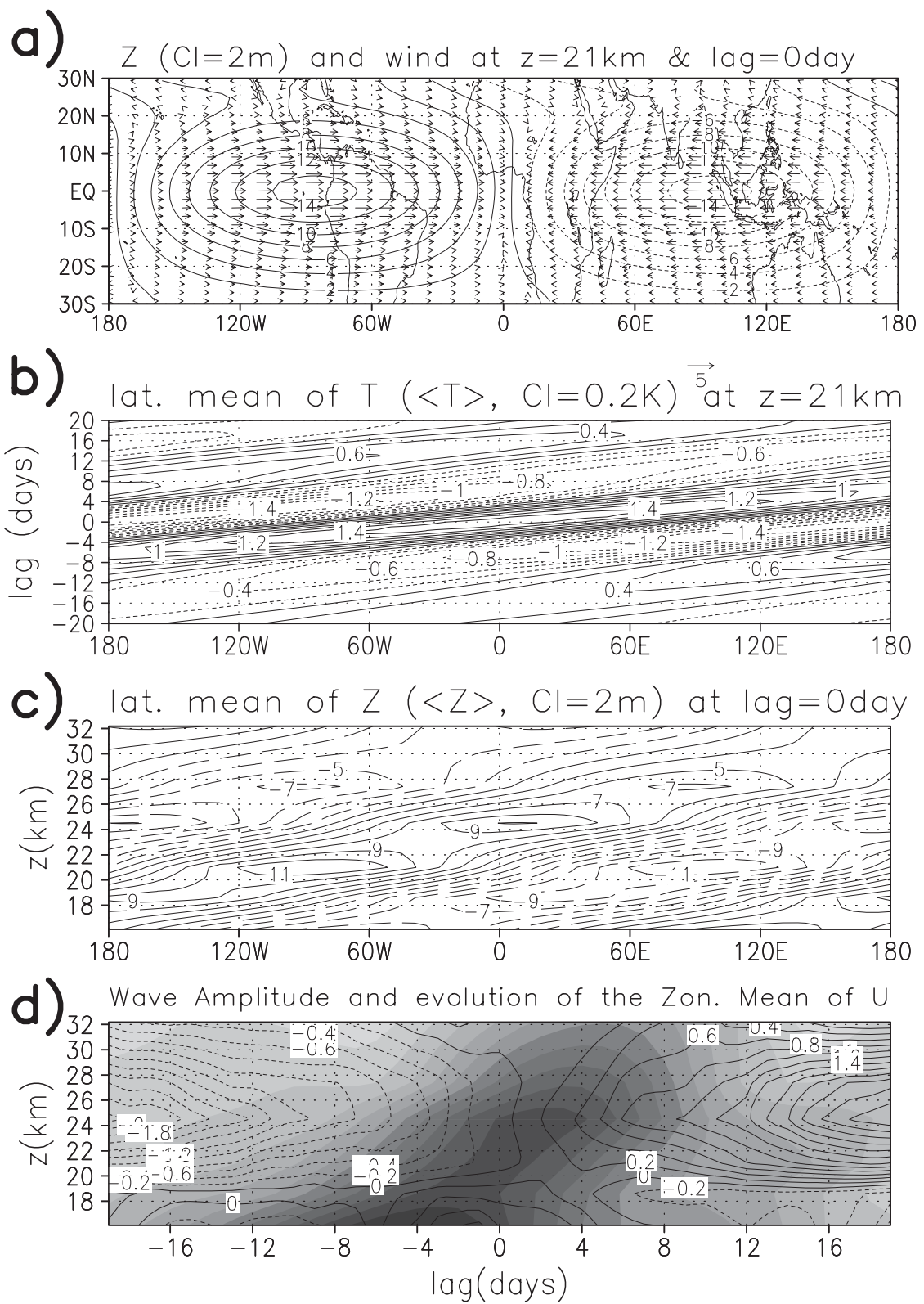

FIG. 7. ERA-40: Composite of the Kelvin waves with wavenumber $s=1$ and periods $\omega^{-1} \approx 10-25$ days (phase speed $C \approx 30 \mathrm{~m} \mathrm{~s}^{-1}$ ). (a) Geopotential and horizontal wind $\left(\tilde{Z}^{c}, \tilde{u}^{c}\right.$, and $\left.\tilde{v}^{c}\right)$ at $z=21 \mathrm{~km}$ and $l=0$ day lag; the geography is arbitrary. (b) Hovmöller diagram of the temperature averaged over the equatorial band $\left\langle\tilde{T}^{c}\right\rangle$ and at $z=21 \mathrm{~km}$. (c) Geopotential averaged over the equatorial band $\left\langle\tilde{Z}^{c}\right\rangle$ at $l=0$ day lag; (d) Wave amplitude measured by $\sqrt{\left\langle\tilde{u}^{c}\right\rangle^{2}}$. CI $=0.2 \mathrm{~m} \mathrm{~s}^{-1}$; contours indicate zonal mean zonal wind evolution [see Eq. (8)].

period is near $\omega^{-1} \approx 16$ days and the signal is much larger in the midlatitudes than in the tropics.

The Hovmöller diagram for the composite $\left\langle\tilde{Z}^{c}\right\rangle$ at $z=21 \mathrm{~km}$ is shown in Fig. 9b. It presents a westwardpropagating disturbance with period $\omega^{-1} \approx 15$ days. Figure 9c presents the vertical section of $\left\langle\tilde{Z}^{c}\right\rangle$ at $l=$
0 day lag. We see that the Rossby wave is almost barotropic and increases with altitude as $e^{2 z / 7 H}$. This last point is confirmed by Fig. 9d, which shows the composite amplitude scaled by $e^{-2 z / 7 H}$ [Eq. (10)]. Figure 9d also shows that the life cycle duration of these Rossby waves is around 25 days. 

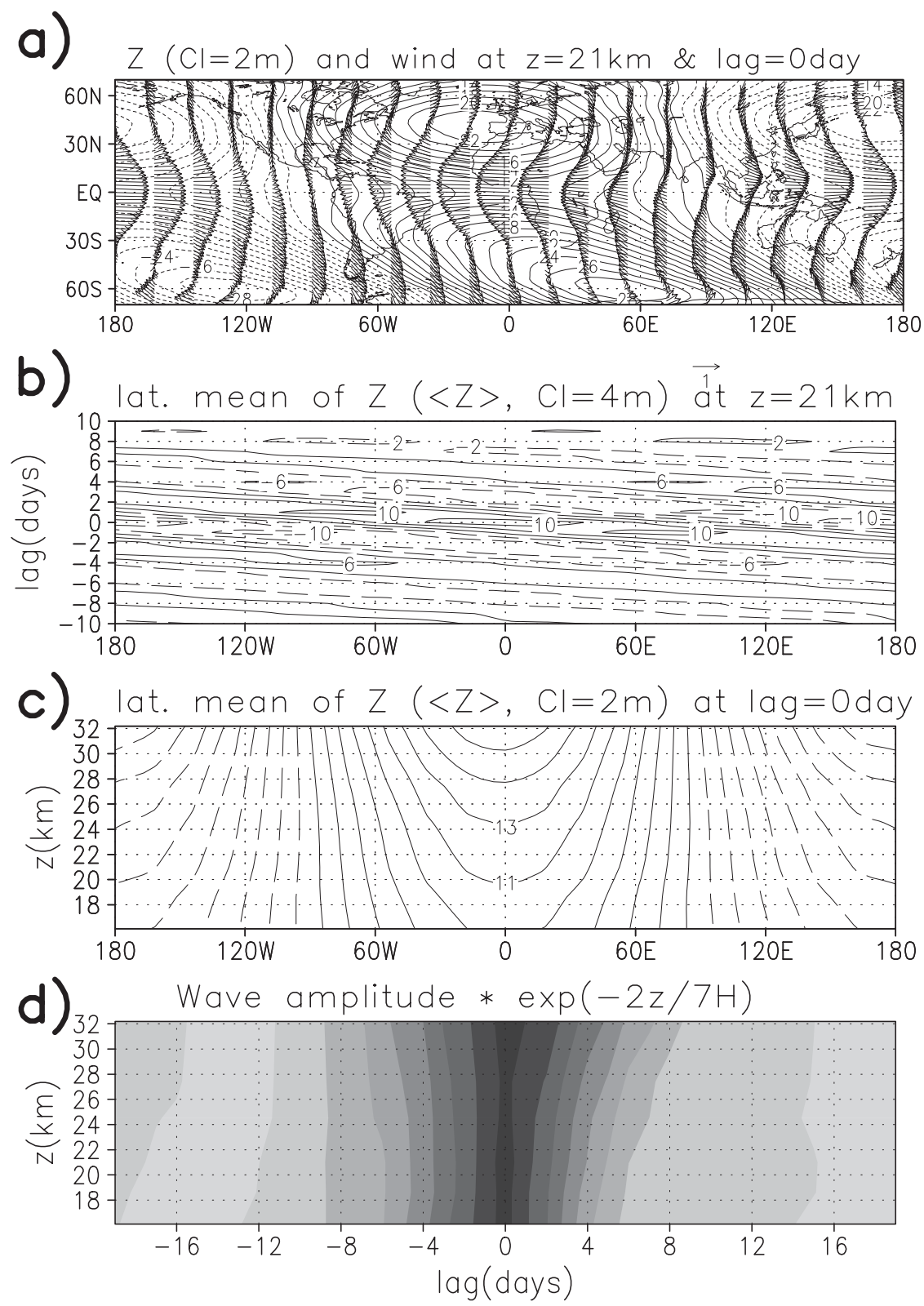

FIG. 8. ERA-40: Composite of the planetary Rossby waves with horizontal wavenumber $s=1$ and periods $\omega^{-1} \approx 5$ days. (a) Geopotential and horizontal wind $\left(\tilde{Z}^{c}, \tilde{u}^{c}\right.$, and $\left.\tilde{v}^{c}\right)$ at $z=21 \mathrm{~km}$ and $l=0$ day lag; the geography is arbitrary. (b) Hovmöller diagram of the geopotential averaged over the equatorial band $\left\langle\tilde{Z}^{c}\right\rangle$ at $z=21 \mathrm{~km}$. (c) Geopotential averaged over the equatorial band $\left\langle\tilde{Z}^{c}\right\rangle$ at $l=0$ day lag. (d) Wave amplitude measured by $\sqrt{\overline{\left\langle\tilde{Z}^{c}\right\rangle^{2}}} e^{-2 z / 7 H}(\mathrm{CI}=1 \mathrm{~m})$.

\section{Results from the LMDz GCM}

\section{a. Model description}

The LMDz GCM is a gridpoint model [Hourdin et al. (2006); its stratospheric version is presented in Lott et al. (2005)]. In this version, the horizontal resolution $\Delta \phi=2.5^{\circ}$ and $\Delta \lambda=3.75^{\circ}$; there are 50 vertical levels, with the upper level at $z=65 \mathrm{~km}$; and the resolution in the lower stratosphere varies slowly from $\Delta z=1 \mathrm{~km}$ at $z=15 \mathrm{~km}$ to $\Delta z=1.8 \mathrm{~km}$ at $z=35 \mathrm{~km}$. The simulation presented here lasts $26 \mathrm{yr}$ and is forced by climatological SSTs and sea ice cover, the first year being used as spinup. It does not have a QBO signal, despite the fact that it uses orographic and nonorographic gravity wave drag schemes (Lott and Miller 1997; Hines 1997). 

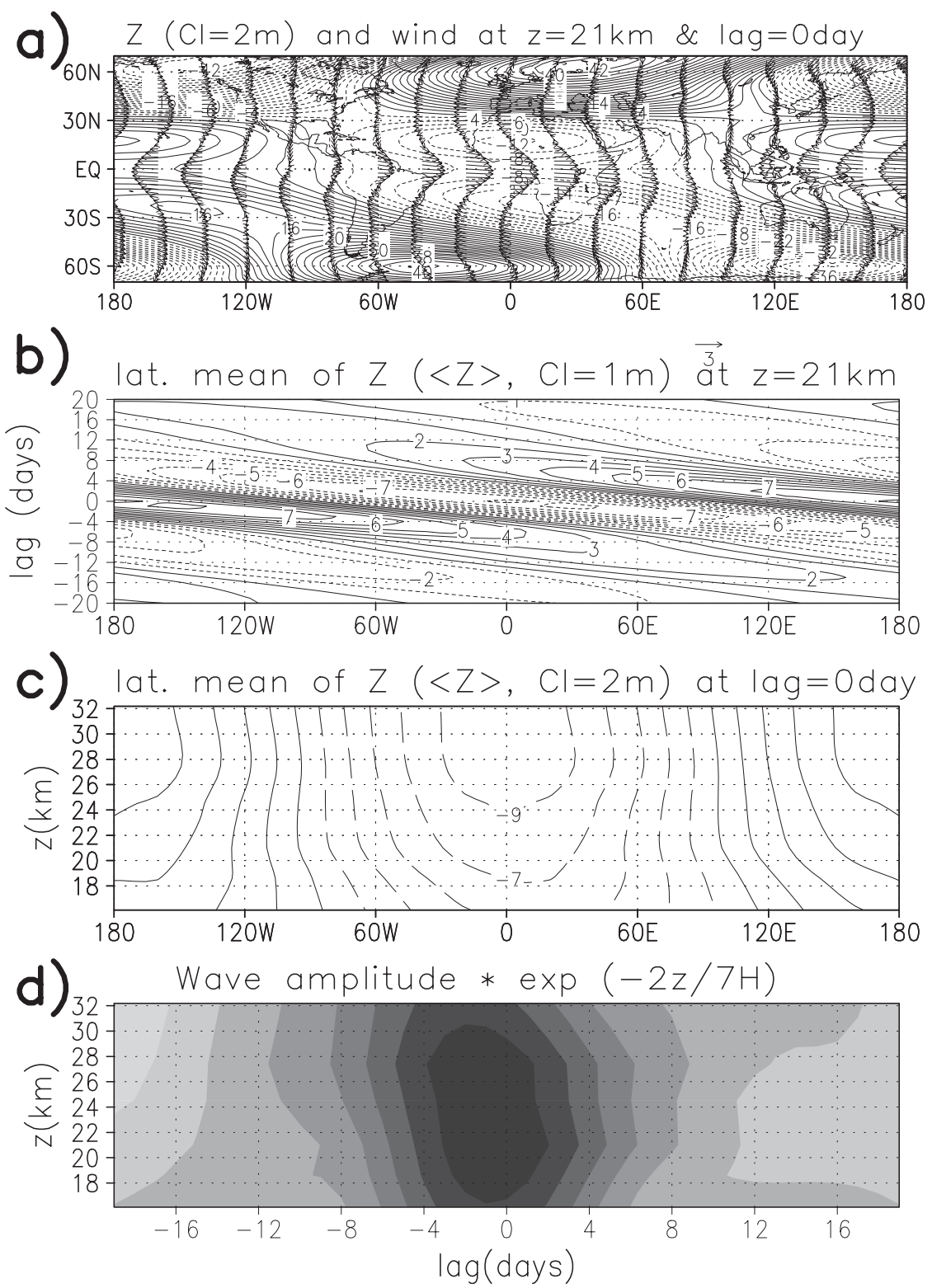

FIG. 9. As in Fig. 8, but for $\omega^{-1} \approx 16$ days, and CI $=0.75 \mathrm{~m}$.

It is also important for the equatorial wave forcing to recall that LMDz uses a convection scheme based on Tiedtke (1989). This is a "mass flux scheme," which requires large-scale moisture convergence (divergence) to trigger (inhibit) deep convection. In this scheme, only one convective cloud is considered in each grid cell, which can yield to large temporal and spatial variability in the simulated precipitations. Horinouchi et al. (2003) have shown that the stratospheric models using this type of scheme simulate more equatorial wave activities than those using parameterizations that do not take into account the large-scale moisture convergence.
To compare the equatorial waves in ERA-40 and in LMDz, it is essential to apply exactly the same procedure to build the composites. More specifically, the spacetime filters and the number of events selected need to be the same, the composite being quite sensitive to both. In this respect, it is also important that the lengths of the datasets be identical ( $25 \mathrm{yr}$ for both) and that the waves analyzed have a comparable spectral signature.

\section{b. Spectral analysis}

The temperature spectrum $S_{\langle T\rangle}$ in Fig. 10a shows a pronounced maximum in the eastward direction for $s=1$ 

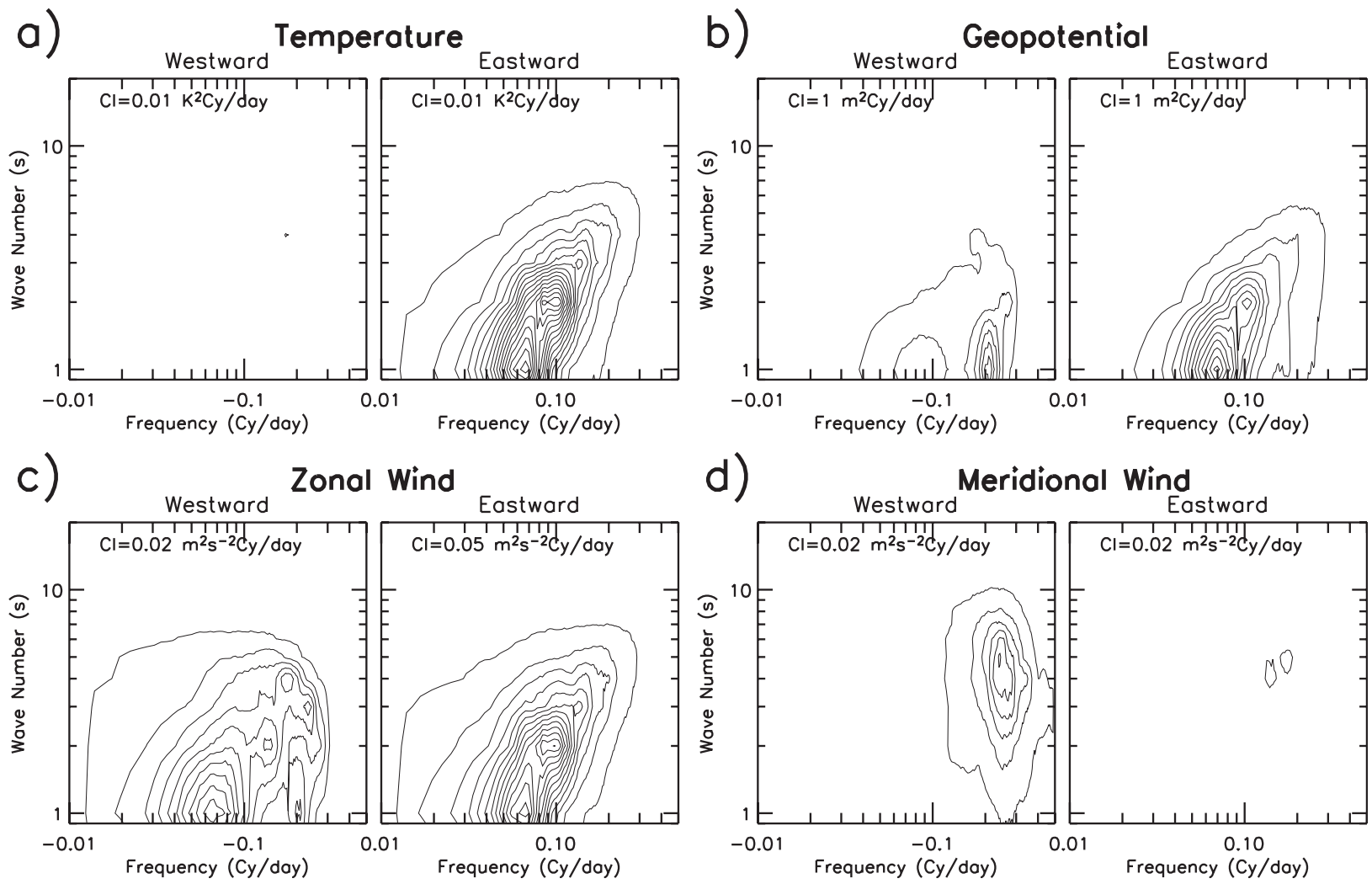

FIG. 10. LMDz: Space-time spectra of fields averaged between $10^{\circ} \mathrm{S}$ and $10^{\circ} \mathrm{N}$ in the lower and middle stratosphere. Each panel displays $s \omega S_{\langle X\rangle}(s, \omega)$ to accommodate the log-scale of the two axis. (a) Temperature $X=T$, (b) geopotential height $X=Z$, (c) zonal wind $X=u$, and (d) meridional wind $X=v$.

and for $\omega^{-1} \approx 10-25$ days, and a secondary maximum for $s>2$ and for $\omega^{-1} \approx 5-15$ days. The first maximum is almost located at the place of the corresponding $s=1$ maximum of the $S_{\langle u\rangle}$ spectra from ERA-40 (Fig. 1a). The second maximum in $\mathrm{LMDz}$ is almost centered on $s=2 \omega^{-1} \approx 10$ days, contrary to ERA-40, and does not extent much to higher frequencies and wavenumbers. Furthermore, the spectral amplitude in LMDz has a larger amplitude than in ERA-40, suggesting that the Kelvin wave signal in the LMDz lower stratosphere is much more pronounced than in the reanalysis. As we will see in the next section, this is due to the fact that the Kelvin waves propagate vertically with much less attenuation in the model, whereas their amplitudes when they enter in the lower stratosphere are comparable to those from the reanalysis.

The spectra for the geopotential and zonal wind in Figs. 10b,c show maxima for $s=1$ at the periodicities of 5 and 16 days in the westward direction that are similar to those from the reanalysis in Figs. 1b,c. It follows that the model simulates the planetary Rossby waves well (not shown), which is due to the fact that its stratospheric variability in the midlatitudes is realistic (Lott et al. 2005). Both spectra also present maxima in the eastward direction associated with the Kelvin waves, as in ERA-40.

The spectrum of the meridional wind in Fig. 10d presents a broad maximum in the westward direction for periods $\omega^{-1} \approx 3-5$ days and wavenumbers $s \approx 2-8$ (i.e., over a narrower temporal band than the corresponding spectrum from ERA-40 in Fig. 1d). As we will see in section $5 \mathrm{~d}$, this is probably due to the fact that the slower Rossby-gravity waves have a vertical wavelength too short to be resolved by the model (the theory predicts $\lambda_{z} \approx 1 \mathrm{~km}$ when $s \approx 4-5$ and $\omega^{-1} \approx 10$ days); the faster ones are easier to reproduce $\left(\lambda_{z} \approx 5 \mathrm{~km}\right.$ when $s \approx 4-5$ and $\omega^{-1} \approx 5$ days).

The half-power point of the filters in Fig. 2 shows that these filters largely capture the relative maxima in the spectra from LMDz (Fig. 10). The resulting composite from $\mathrm{LMDz}$ can therefore be compared to those from ERA-40. For the planetary Rossby waves, we found that the spatiotemporal composites in $\mathrm{LMDz}$ are similar to those in the reanalysis (not shown). We also found that $\mathrm{LMDz}$ produces an $s=1$ Kelvin wave which is almost like that in the reanalysis, at least at the key altitude 

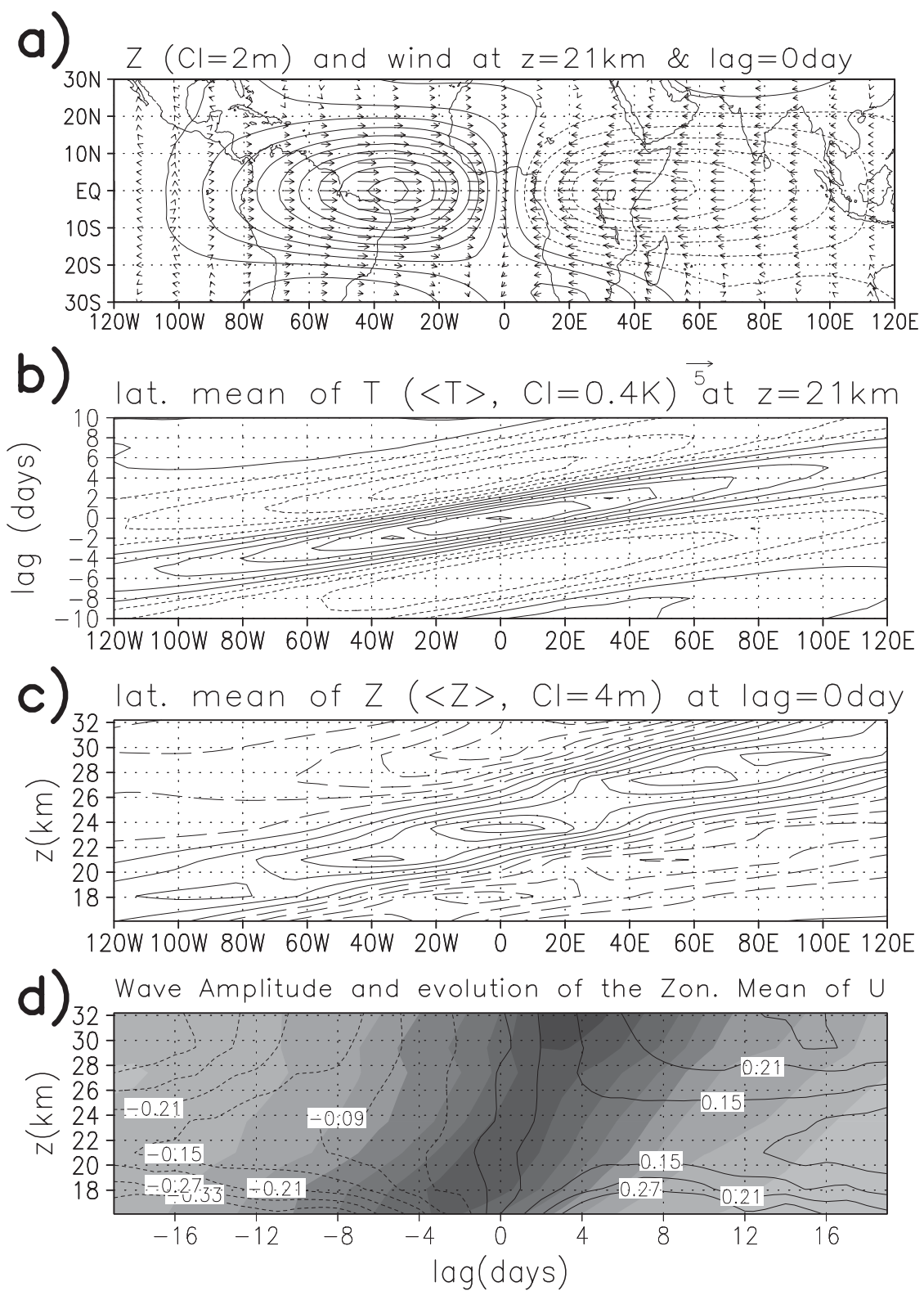

FIG. 11. LMDz: composite of the Kelvin waves with wavenumbers $s=2-5$ and periods $\omega^{-1} \approx 3-10$ days (phase speed $C \approx 25 \mathrm{~m} \mathrm{~s}^{-1}$ ). (a) Geopotential and horizontal wind $\left(\tilde{Z}^{c}, \tilde{u}^{c}\right.$, and $\tilde{v}^{c}$ ) at $z=21 \mathrm{~km}$ and $l=0$ day lag; the geography is arbitrary. b) Hovmöller diagram of the temperature averaged over the equatorial band $\left\langle\tilde{T}^{c}\right\rangle$ at $z=21 \mathrm{~km}$. (c) Geopotential height averaged over the equatorial band $\left\langle\tilde{Z}^{c}\right\rangle$ and at $l=0$ day lag. (d) Wave amplitude measured by $\sqrt{\left\langle\tilde{u}^{c}\right\rangle^{2}}$. CI $=$ $0.2 \mathrm{~m} \mathrm{~s}^{-1}$; contours indicate zonal mean zonal wind evolution [see Eq. (8)].

$z=21 \mathrm{~km}$ and below (not shown). At higher levels it is much less attenuated in the model than in the reanalysis; this effect will be discussed for the Kelvin wave packets in the next section.

\section{c. Kelvin wave packets}

The LMDz composites for the Kelvin wave packets at $z=21 \mathrm{~km}$ and $l=0$ day lag in Fig. 11a) compare well with those from ERA-40 in Fig. 4a, indicating that LMDz has Kelvin waves with the correct amplitude in the lower stratosphere. Nevertheless, the zonal extension of the composite in $\mathrm{LMDz}$ is much larger than in ERA-40. This indicates that LMDz underestimates the relatively large wavenumbers (e.g., $s=3,4$ ). Note that this deficit was already apparent in the LMDz spectra in Figs. 10a-c: their eastward maximum falls off quite 
rapidly in amplitude for the zonal wavenumbers $s \geq 3$, whereas in ERA-40 these eastward maxima extend up to $s=5,6$.

The Hovmöller diagram for the temperature $\langle\tilde{T}\rangle^{c}$ in Fig. $11 \mathrm{~b}$ shows eastward phase propagation and eastward group velocity as expected. The period is around $\omega^{-1} \approx 10$ days and is longer than the period found in ERA-40 $\left(\omega^{-1} \approx 7\right.$ days $)$. Again, this is consistent with the spectral estimates in Figs. 10a-c, which have a relatively small amplitude in the eastward direction for $\omega^{-1} \leq$ 6-7 days, when compared to ERA-40 in Figs. 1a-c.

The longitude-altitude section of $\langle\tilde{Z}\rangle^{c}$ at $l=0$ day lag in Fig. 11c is positively tilted with altitude, indicating upward propagation. The vertical wavenumber is around $\lambda_{z} \approx 13 \mathrm{~km}$. It is significantly larger than the theoretical value (theory predicts $\lambda_{z} \approx 7 \mathrm{~km}$, when $s=2$, and $\omega^{-1}=10$ days). This effect is due to the fact that the zonal wind is negative in the lower stratosphere of LMDz (around and below $\bar{u}=-10 \mathrm{~m} \mathrm{~s}^{-1}$; not shown), yielding to shorter intrinsic periods and larger vertical wavelengths.

In Figs. 11c,d [note that in Fig. 11d the shaded contours are for the composite amplitude; see Eq. (7)], we see that the Kelvin wave amplitude is almost constant with altitude. The Kelvin waves in LMDz are therefore much less attenuated than in ERA-40 (see Figs. 4c,d). Nevertheless, some attenuation occurs in LMDz as well because the amplitude of the equatorial waves varies as $e^{z / 2 H}$ in the nondissipative case, whereas here it is almost constant.

Another indication that the waves entering in the composites are attenuated is provided by the composite of the zonal mean zonal wind evolution during the life cycle of the Kelvin waves [see Eq. (8) and the black lines in Fig. 4d]. It shows that the zonal mean zonal wind increases by around $0.2-0.3 \mathrm{~m} \mathrm{~s}^{-1}$ during the life cycle of the Kelvin waves, consistent with the picture that these waves accelerate the zonal mean flow when they are dissipated. Interestingly, in ERA-40 we have seen that the increase of the zonal wind during the Kelvin wave life cycles can in part be due to a sampling of the QBO signal. Because LMDz does not have a QBO signal, the result from $\mathrm{LMDz}$ is a therefore a better illustration of the zonal mean zonal wind acceleration produced by the Kelvin waves dissipation.

\section{d. Rossby-gravity waves $(s \geq 2)$}

The composite maps in Fig. 12 show that LMDz has difficulties in simulating the Rossby-gravity waves. The composite patterns for $Z, u$, and $v$ at $l=0$ day lag and $z=21 \mathrm{~km}$ in Fig. 12a have almost half the amplitude of those from ERA-40 in Fig. 6a (note the changes in scales between the two figures). The equatorial antisymmetry of the geopotential height and of the zonal wind that characterizes Rossby-gravity waves is in part lost. Note, however, that the composite is dominated by the wavenumbers $s=5,6$ which is consistent with the results from ERA-40.

At $z=21 \mathrm{~km}$, the Hovmöller diagram for $\langle\tilde{v}\rangle^{c}$ in Fig. $12 \mathrm{~b}$ is more irregular than in ERA-40 and shows that the characteristic period of the Rossby-gravity waves in LMDz is around $\omega^{-1} \approx 4$ days; that is, 2 days shorter than in ERA-40. It also shows westward phase propagation, but the phase speed is larger in amplitude than in ERA-40 $\left(C \approx-25 \mathrm{~m} \mathrm{~s}^{-1}\right.$ in LMDz versus $-15 \mathrm{~m} \mathrm{~s}^{-1}$ in ERA-40). Figure $12 \mathrm{~b}$ also shows that the composite patterns are more stationary in amplitude than in ERA-40. This is due to the fact that the positive intrinsic group velocity of the Rossby-gravity waves is balanced here by the negative zonal mean zonal winds that are dominant in the lower equatorial stratosphere of LMDz.

The longitude-altitude section of $\langle\tilde{v}\rangle^{c}$ at $l=0$ day lag in Fig. 12c shows that the lines of constant phase are negatively tilted, indicating upward propagation. The vertical wavelength is around $\lambda_{z} \approx 6 \mathrm{~km}$ and is almost 2 times smaller than predicted by theory. Again, it follows that the zonal mean zonal wind in the LMDZ equatorial stratosphere is negative, which tends to reduce the intrinsic frequency and the vertical wavelength of the waves.

The time-altitude diagram for the amplitude [see Eq. (9)] in Fig. 12d shows that the Rossby-gravity waves in LMDz have a life cycle of 10-15 days, and they never extend above $25 \mathrm{~km}$. At this altitude, the zonal mean zonal wind $\bar{u}$ is around $-15 \mathrm{~m} \mathrm{~s}^{-1}$ in LMDz (not shown) - that is, close to critical-level condition for the Rossby-gravity waves here, their absolute phase velocity being around $C_{a} \approx-25 \mathrm{~m} \mathrm{~s}^{-1}$. Furthermore, for an intrinsic phase velocity of $C_{i}=C_{a}-\bar{u}=-10 \mathrm{~m} \mathrm{~s}^{-1}$, the vertical wavelength of the $s=5$ and $s=6$ waves becomes smaller than $\lambda_{z}=1 \mathrm{~km}$ (e.g., smaller than the vertical resolution of $\mathrm{LMDz}$ ). We can therefore conclude that the Rossby-gravity waves in the model are dissipated numerically as they propagate upward. This process results in a deceleration of $\bar{u}$, as shown by the composite for the evolution of the zonal wind [see Eq. (8) and the contour lines in Fig. 12d). Again, because the model does not have a QBO, this is a better illustration of the zonal mean deceleration produced by Rossby-gravity waves than in ERA-40, but the amplitude of the deceleration is also much smaller.

\section{Conclusions}

\section{a. Summary}

In the lower stratosphere, the gravest equatorial waves are the Kelvin and the Rossby-gravity waves. They are 


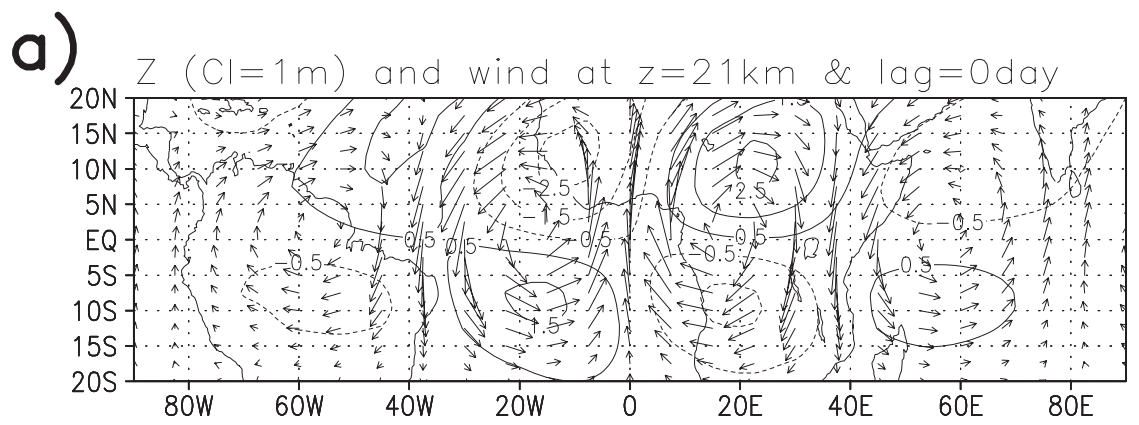

b) Latitude Mean of $V(C l=1 \mathrm{~m} / \mathrm{s})$ at $\stackrel{2}{\mathrm{z}=21 \mathrm{~km}}$
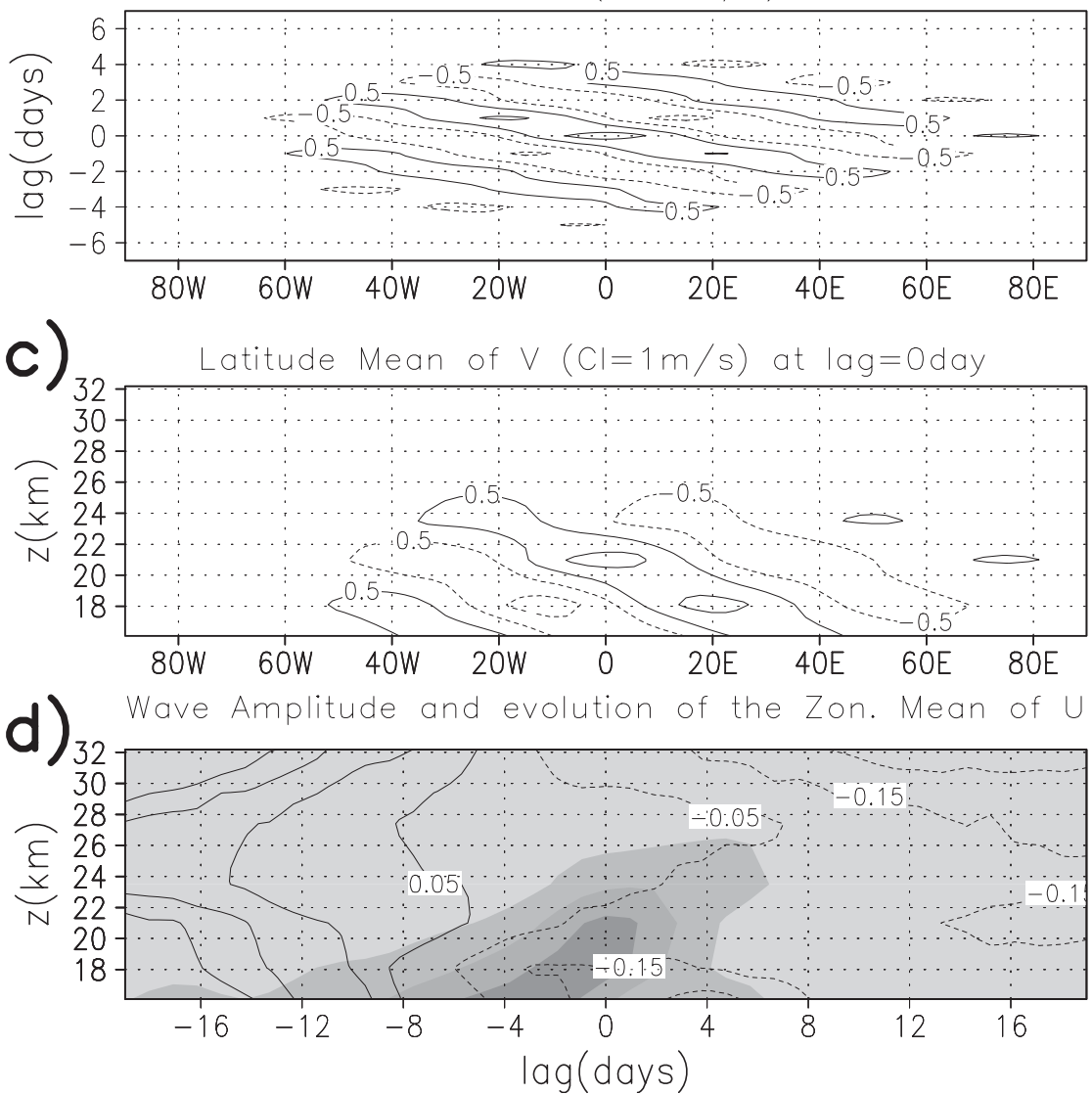

FIG. 12. LMDz: composite of the Rossby-gravity waves with wavenumbers $s=4-8$ and periods $\omega^{-1} \approx 3-8$ days (phase speed $C \approx-25 \mathrm{~m} \mathrm{~s}^{-1}$. (a) Geopotential and horizontal wind $\left(\tilde{Z}^{c}, \tilde{u}^{c}\right.$, and $\left.\tilde{v}^{c}\right)$ at $z=21 \mathrm{~km}$ and $l=0$ day lag; the geography is arbitrary. (b) Meridional wind averaged over the equatorial band $\left\langle\tilde{v}^{c}\right\rangle$ at $z=21 \mathrm{~km}$. (c) Meridional wind averaged over the equatorial band $\left\langle\tilde{v}^{c}\right\rangle$ at $l=0$ day lag. (d) Wave amplitude measured by $\sqrt{\left.\overline{\left\langle\tilde{v}^{c}\right.}\right\rangle^{2}}$. CI $=0.2 \mathrm{~m} \mathrm{~s}^{-1}$; contours indicate zonal mean zonal wind evolution [see Eq. (8)].

often grouped in packets in the horizontal direction (see the examples in Figs. $3 b$ and $5 b$ ) because they are just above their tropospheric sources. It is within these packets that irreversible processes, such as breaking or condensation of ice, are triggered. The method presented here extracts from ERA-40 a climatology of these wave packets. It also extracts a climatology of the equatorial signal produced by some planetary Rossby waves, which we found to be quite substantial in the equatorial regions. The method presented has four advantages. The first is that it extracts the signal due to each type of wave on all the dynamical fields $(u, v, Z$, and $T$ ). The second is that it gives a description of these signals in terms of packets, yielding to composites for 
which the horizontal extension, life cycle duration, and amplitude compare well with those seen in particular cases (for the Kelvin wave packets, compare Figs. 3b and $4 \mathrm{~b}$; for the Rossby-gravity waves, compare Figs. $5 \mathrm{~b}$ and $6 \mathrm{~b}$ ). The third is that for all the waves presented, the signal induced on the dynamical fields is always significant and is quite large: it compares with the standard deviation of the corresponding fields evaluated over the equatorial band (see Table 2). The fourth is that our composites show the waves entering in the lower stratosphere, propagating vertically through it, being dissipated, and producing zonal mean zonal flow changes.

For the Kelvin waves, the dominant packets are constituted of harmonics with wavenumbers $s \approx 2-5$ and periods around 7 days (Fig. 7). These wave packets have a characteristic life cycle that lasts around 15-20 days and are dissipated in the lower stratosphere. An increase of the zonal wind in the lower stratosphere is also observed when these packets propagate through it, which is consistent with the pictures that these Kelvin wave packets contribute to the easterly phase of the QBO. For the Rossby-gravity waves, the dominant packets are constituted of harmonics with wavenumbers $s \approx 3-9$ and periods around 5-6 days (Fig. 6). These wave packets have a life cycle that lasts around 10-15 days and are dissipated in the lower stratosphere. A decrease of the zonal wind in the lower stratosphere is also observed, which is consistent with the pictures that these Rossby-gravity wave packets contribute to the easterly phase of the QBO.

Another strength of the method is that it can be used to isolate the life cycle of individual zonal harmonics. This approach is mandatory to describe the equatorial signature of the free planetary waves, which we found to be very substantial on the geopotential height and on the horizontal wind, at least for the $s=1$ signals (see Figs. 8 and 9 for the 5-day and 16-day waves, respectively). This approach is also useful for the Kelvin waves because there are periods of times when the $s=1$ Kelvin waves are very strong (Fig. 7).

We have also shown that this method can be applied to GCMs to quantify how well they simulate the equatorially trapped and the planetary Rossby waves. When applied to $\mathrm{LMDz}$, this method shows that the model simulates Kelvin waves with reasonable amplitude in the lower stratosphere (Fig. 11). These waves are much less dissipated than in ERA-40, but this is due to the fact that LMDz does have a QBO. In the model, the Kelvin wave propagation in the lower stratosphere is also accompanied by an increase of the zonal wind, illustrating that Kelvin waves accelerate the zonal mean zonal flow in the lower stratosphere. Note, however, that LMDz fails to simulate the Kelvin waves with periods around $\omega^{-1} \approx 7$ days and zonal wavenumbers larger than $s=3$. Because these waves have a relatively large vertical wavenumber (on the order of $\lambda_{z} \approx 10 \mathrm{~km}$ ), this error probably follows from an insufficient tropospheric forcing at the horizontal wavenumbers $s>3$.

$\mathrm{LMDz}$ also has some Rossby-gravity waves, which decelerate the zonal mean zonal wind. These Rossbygravity waves nevertheless do not project very well on the theoretical structure of these waves and are rapidly attenuated in the vertical direction (Fig. 12). This signal is also very weak for periods larger than $\omega^{-1} \approx 5$ days. These defects are due to the fact that the Rossbygravity waves with periods around 10 days have a vertical wavenumber $\lambda_{z} \leq 1 \mathrm{~km}$ and thus cannot be handled by a model with vertical resolution larger than that. On top of this, the zonal mean zonal winds in the model lower stratosphere are predominantly easterlies; this tends to decrease the intrinsic frequency of the westerly waves and their vertical wavenumber. If we recall now that ERA-40 also underestimates the Rossby-gravity waves (Ern et al. 2008), we can conclude here that LMDz fails to simulate a good part of them.

To summarize the results from LMDz, this analysis shows that its defects have two different causes. The first is that it has a too coarse vertical resolution, which is crucial for the Rossby-gravity waves, and the second is that it has an insufficient tropospheric forcing of the waves with wavenumbers $s>3$, as was identified for the Kelvin waves. Therefore, this analysis calls for further model sensitivity studies to the vertical resolution and to the convection schemes. These are beyond the scope of this paper, but the analysis presented here clearly demonstrates the strength of the method to validate model calculations.

\section{b. On the relations with the $Q B O$}

The relationships between the gravest equatorial waves and the zonal wind changes found in ERA-40 and in LMDz illustrate how these waves can contribute to the QBO (for the Kelvin wave life cycles and the zonal mean flow evolutions, see Figs. 4d, 7d, and 11d; for the Rossby-gravity waves and the zonal mean flow evolutions, see Figs. $6 \mathrm{~d}$ and 12d). Nevertheless, the accelerations due to the Kelvin waves and the decelerations due to the Rossby-gravity waves seen in ERA-40 (Figs. $4 \mathrm{~d}$ and $7 \mathrm{~d}$, respectively) are much larger in amplitude then those seen in LMDz (Figs. 11d and 12d, respectively). These differences in amplitude can have two causes. The first is that the equatorial waves in $\mathrm{LMDz}$ are too small; the second is that in ERA-40 the composite analysis samples the QBO signal differently during the periods when the Kelvin waves are extracted and 
when the Rossby-gravity waves are extracted. This second effect is quite important. In fact, we have also found that the Kelvin waves are larger when the zonal mean zonal winds are negative at the altitude where we detect the waves ( $z=21 \mathrm{~km}$; Fig. 3a). Accordingly, for the dates when we extract the waves, the QBO is in general increasing aloft $z=21 \mathrm{~km}$. The opposite is true for the Rossby-gravity waves (we extract them when the zonal wind is positive at $z=21 \mathrm{~km}$ so the QBO is decreasing aloft). This means that even if these waves do not produce substantial changes in the zonal winds, we would observe those changes using our method.

We have tried to reduce this effect in ERA-40 by applying a high-pass temporal filter to the zonal mean zonal wind. With a filter that keeps the period $\omega^{-1}<2$ months, the QBO signal is suppressed but the zonal wind variations occurring on the time scale of the life cycle of our waves are unaffected. With this method, preliminary results show that the amplitudes of the zonal mean flow evolutions due to those waves in ERA-40 are larger than in LMDz, but not as much as was shown here: it seems that the acceleration from LMDz in Figs. $11 d$ and $12 d$ is quite representative of that produced by these waves in reality. These preliminary results suggest that the gravest equatorial waves only produce a fraction of the QBO signal. This requires that the analysis be extended to higher frequencies and higher wavenumbers.

Acknowledgments. We would like to acknowledge Dr G. Kiladis and two anonymous referees for their helpful comments that improved this paper.

\section{REFERENCES}

Baldwin, M. P., and Coauthors, 2001: The quasi-biennial oscillation. Rev. Geophys., 39, 179-229.

Boville, B. A., and W. J. Randel, 1992: Equatorial waves in a stratospheric GCM: Effects of vertical resolution. J. Atmos. Sci., 49, 785-801.

Dunkerton, T. J., and M. P. Baldwin, 1995: Observation of 3-6-day meridional wind oscillations over the tropical Pacific, 1973-1992: Horizontal structure and propagation. J. Atmos. Sci., 52, 1585-1601.

Ern, M., P. Preusse, M. Krebsbach, M. G. Mlynczak, and J. M. Russell III, 2008: Equatorial wave analysis from SABER and ECMWF temperatures. Atmos. Chem. Phys., 8, 845-869.

Fujiwara, M., F. Hasebe, M. Shiotani, N. Nishi, H. Voemel, and S. J. Oltmans, 2001: Water vapor control at the tropopause by equatorial Kelvin waves observed over the Galapagos. Geophys. Res. Lett., 28, 3143-3146.

—- M. K. Yamamoto, H. Hashiguchi, T. Horinouchi, and S. Fukao, 2003: Turbulence at the tropopause due to breaking Kelvin waves observed by the Equatorial Atmosphere Radar. Geophys. Res. Lett., 30, 1171, doi:10.1029/2002GL016278.

Giorgetta, M. A., E. Manzini, E. Roeckner, M. Esch, and L. Bengston, 2006: Climatology and forcing of the quasi-biennial oscillation in the MECHAM5 model. J. Climate, 19, 3882-3901.

Hendon, H. H., and M. C. Wheeler, 2008: Some space-time spectral analyses of tropical convection and planetary-scale waves. J. Atmos. Sci., 65, 2936-2948.

Hertzog, A., and F. Vial, 2001: A study of the dynamics of the equatorial lower stratosphere by use of ultra-long-duration balloons. 2. Gravity waves. J. Geophys. Res., 106, 22 745-22 761.

Hines, C. O., 1997: Doppler spread parameterization of gravity wave momentum deposit in the middle atmosphere. Part 1: Basic formulation. J. Atmos. Terr. Phys., 59, 371-386.

Hirota, I., and T. Hirooka, 1984: Normal mode Rossby waves observed in the upper stratosphere and mesosphere. Part I: First symmetric modes of zonal wavenumbers 1 and 2. $J$. Atmos. Sci., 41, 1253-1267.

Holton, J. R., 1972: Waves in the equatorial stratosphere generated by tropospheric heat sources. J. Atmos. Sci., 29, 368-375. , and R. S. Lindzen, 1972: An updated theory for the quasibiennial cycle of the tropical stratosphere. J. Atmos. Sci., 29, 1076-1107.

Horinouchi, T., and Coauthors, 2003: Tropical cumulus convection and upward-propagating waves in middle-atmospheric GCMs. J. Atmos. Sci., 60, 2765-2782.

Hourdin, F., and Coauthors, 2006: The LMDZ4 general circulation model: Climate performance and sensitivity to parametrized physics with emphasis on tropical convection. Climate Dyn., 27, 787-813, doi:10.1007/s00382-006-0158-0.

Jensen, E. J., L. Pfister, A. S. Ackerman, A. Tabazadeh, and O. B. Toon, 2001: A conceptual model of the dehydration of air due to freeze-drying by optically thin, laminar cirrus rising slowly across the tropical tropopause. J. Geophys. Res., 106, 17 237-17 252.

Lott, F., and M. J. Miller, 1997: A new subgrid-scale orographic drag parameterization: Its formulation and testing. Quart. J. Roy. Meteor. Soc., 123, 101-127.

, L. Fairhead, F. Hourdin, and P. Levan, 2005: The stratospheric version of LMDz: Dynamical climatologies, Arctic oscillation, and impact on the surface climate. Climate Dyn., 25, 851-868, doi:10.1007/s00382-005-0064-x.

Madden, R. A., 1978: Further evidence of traveling planetary waves. J. Atmos. Sci., 35, 1605-1618.

_ 2007: Large-scale free Rossby waves in the atmosphere-An update. Tellus, 59A, 571-590.

Manzini, E., and K. Hamilton, 1993: Middle atmospheric traveling waves forced by latent and convective heating. J. Atmos. Sci., 50, 2180-2200

Matsuno, T., 1966: Quasi-geostrophic motions in the equatorial area. J. Meteor. Soc. Japan, 44, 25-43.

Matthews, A. J., 2000: Propagation mechanism for the MaddenJulian oscillation. Quart. J. Roy. Meteor. Soc., 126, 2637-2652.

Mote, P. W., and T. J. Dunkerton, 2004: Kelvin wave signatures in stratospheric trace constituents. J. Geophys. Res., 109, D03101, doi:10.1029/2002JD003370.

,-- , and D. Wu, 2002: Kelvin waves in stratospheric temperature observed by the Microwave Limb Sounder. J. Geophys. Res., 107, 4218, doi:10.1029/2001JD001056.

Randel, W. J., and J. C. Gille, 1991: Kelvin wave variability in the upper stratosphere observed in SBUV ozone data. J. Atmos. Sci., 48, 2330-2349.

, and F. Wu, 2005: Kelvin wave variability near the equatorial tropopause observed in GPS radio occultation measurements. J. Geophys. Res., 110, D03102, doi:10.1029/2004JD005006.

Rodgers, C. D., 1976: Evidence for the five-day wave in the upper stratosphere. J. Atmos. Sci., 33, 710-711. 
Salby, M. L., 1981: Rossby normal modes in nonuniform background configurations. Part II: Equinox and solstice conditions. J. Atmos. Sci., 38, 1827-1840.

Sasi, M. N., and Coauthors, 2003: A study of equatorial waves characteristics using rockets, balloons, lidar and radar. $A d v$. Space Res., 32, 813-818, doi:10.1016/S0273-1177(03)00412-5.

Shiotani, M., J. G. Gille, and A. E. Roche, 1997: Kelvin waves in the equatorial lower stratosphere as revealed by cryogenic limb array etalon spectrometer temperature data. J. Geophys. Res., 102 (D22), 26 131-26 140.

Tiedtke, M., 1989: A comprehensive mass flux scheme for cumulus parameterization in large-scale models. Mon. Wea. Rev., 117, 1779-1800.

Tindall, J. C., J. Thuburn, and E. J. Highwood, 2006a: Equatorial waves in the lower stratosphere. I: A novel detection method. Quart. J. Roy. Meteor. Soc., 132, 177-194.

- - , and,$- 2006 \mathrm{~b}$ : Equatorial waves in the lower stratosphere. II: Annual and interannual variability. Quart. J. Roy. Meteor. Soc., 132, 195-212.

Tsuda, T., Y. Murayama, H. Wiryosumarto, S. W. B. Harijono, and S. Kato, 1994: Radiosonde observations of equatorial atmos- phere dynamics over Indonesia. 1. Equatorial waves and diurnal tides. J. Geophys. Res., 99, 10 491-10 505.

Uppala, S. M., and Coauthors, 2005: The ERA-40 Re-Analysis. Quart. J. Roy. Meteor. Soc., 131, 2961-3012, doi:10.1256/ qj.04.176.

Vial, F., A. Hertzog, C. R. Mechoso, C. Basdevant, P. Cocquerez, V. Dubourg, and F. Nouel, 2001: A study of the dynamics of the equatorial lower stratosphere by use of ultra-longduration balloons. 1: Planetary scales. J. Geophys. Res., 106, $22725-22743$.

Wallace, J. M., and V. E. Kousky, 1968: Observational evidence of Kelvin waves in the tropical stratosphere. J. Atmos. Sci., 25, 900-907.

Wheeler, M., G. N. Kiladis, and P. J. Webster, 2000: Large-scale dynamical fields associated with convectively coupled equatorial waves. J. Atmos. Sci., 57, 613-640.

Yanai, M., and T. Maruyama, 1966: Stratospheric wave disturbances in the tropical stratosphere. J. Meteor. Soc. Japan, 44, 291-294.

Yang, G.-Y., B. Hoskins, and J. Slingo, 2003: Convectively coupled equatorial waves: A new methodology for identifying wave structures in observational data. J. Atmos. Sci., 60, 1637-1654. 